\title{
Real-time optimization-based reference calculation integrated control for MMCs considering converter limitations
}

\author{
Daniel Westerman Spier, Graduate Student Member, IEEE, Joan-Marc Rodriguez-Bernuz, Member, IEEE, \\ Eduardo Prieto-Araujo, Senior Member, IEEE, Joaquim López-Mestre \\ Adrià Junyent-Ferré, Senior Member, IEEE, and Oriol Gomis-Bellmunt, Fellow, IEEE
}

\begin{abstract}
The paper addresses a real-time optimization-based reference calculation integrated with a control structure for Modular Multilevel Converters (MMC) operating under normal and constrained situations (where it has reached current and/or voltage limitations, as it may occur during system faults). Firstly, a nonlinear optimization problem has been developed in which it prioritizes to satisfy the AC grid current set-points imposed by the transmission System Operator (TSO). The constrained nonlinear optimization problem is formulated based on the steady-state model of the MMC, whereby the prioritization is achieved through distinct weights defined in the Objective Function's (OF) terms. The resultant optimization problem, however, is highly nonlinear requiring high computation burden to be solved in real-time. To cope with this issue, this paper applies a Linear Time-Varying (LTV) approximation, which permits to represent the nonlinear dynamics of the system as constant parameters, while a Linear Time-Invariant (LTI) system is used to formulate the optimization constraints. The converter's current references are determined in real-time by solving a constrained linearized optimization problem at each control time step, which considers the TSO's demands, the current MMC operating point and its physical limitations. Theoretical analyses comparing the responses of the linear and nonlinear optimization problems are performed to validate the accuracy of the LTV approximation. Finally, the linearized-optimization problem is integrated with the MMC controllers, evaluated under different AC and DC network conditions and compared with conventional control strategies, where it is shown that the presented method can be potentially employed to obtain the MMC current references for distinct network scenarios.
\end{abstract}

Index Terms-Modular multilevel converter (MMC), real-time optimization reference calculation, real-time saturations, reactive power control.

\section{INTRODUCTION}

A MONG the different topologies of Voltage Source Converters for High Voltage Direct Current (VSC-HVDC), the MMC has emerged as the preferred choice due its superior efficiency, easier voltage scalability and reduced AC output voltage harmonics [1], [2]. Compared to classical two- and three-level VSCs, MMCs, on the one hand, allows the powers from the AC and DC sides to be controlled independently [3]. On the other hand, it presents an important increment in the number of degrees of freedom that must be considered during the modelling and control stages in order to maintain the proper operation of the converter, especially during $\mathrm{AC}$ and DC network voltage unbalances [4].

Industrial practices given by Cigre suggest that the MMC's controllers should regulate the AC network currents, the circulating currents and the internal energy of the converter [5]. Under balanced grid conditions, traditional control strategies such as the circulating current suppressing control (CCSC) [6] can be used to keep the MMC's quantities close to their nominal values. But under scenarios where energy deviations occur for instance, during $\mathrm{AC} / \mathrm{DC}$ power set-points changes, the CCSC may present lower performance compared to strategies that regulate the MMC's SM capacitors energies [7]. Nevertheless, during unbalanced AC and/or DC faults, several quantities of the converter are importantly affected. The internal states of the converter must be properly compensated in order to maintain the system operating accordingly to the network operator requirements (e.g. providing frequency or voltage support to the grid) without transgressing the physical limitations of the converter.

Previous works in the research literature have addressed the modelling and control of the MMC in order to mitigate network fault effects [8]. The CCSC strategy has been improved in [9], but still the internal energy balancing of the converter is disregarded. To overcome such issue, different control methods are proposed in [10]-[15]. Authors in [10] employed a multi-hierarchy control strategy targeting the energy mismatch between the upper and lower arms of the MMC. Similarly, [11], [12] analysed the energy unbalance among the phaselegs of the converter. Although the previous works have merits, there is still a need to combine their approaches to consider both phase-legs' and upper and lower arms' energy disparity. Reference [14] addressed this matter, but it disregarded the influence of the negative-sequence current component circulating through the converter during unbalanced faults. Differently, [15] included such current component in its control strategy, but neglected the effects of the AC network and the arm impedances in order to simplify the mathematical derivations.

Focusing on enhancing the performance of the MMC, several authors employed optimization algorithms as part of the control strategy. By doing so, the internal energy and circulating current limitations of the converter can be easily imposed in the formulation of the problem. In [16], [17], different optimization problems are developed targeting the internal energy balancing and optimizing the circulating currents, which are later used in the control strategy. However, due to their high nonlinear characteristics (resulting in high computational burden), these models have to be used in an offline manner. Thus, the control variables required from the optimal algorithm are calculated prior their application into the real system. Further improvements can be achieved if the optimization problem can be solved in real-time. In [18], a real-time linear matrix inequality optimization problem is 
integrated with conventional $\mathrm{AC}$ grid and internal energy control strategies. But, the controller cannot compensate the MMC's internal energy producing undesired sustained deviations during unbalance AC faults. Model Predict Control (MPC) is also a potential candidate to optimize the MMC's operation. In [19], [20], different MPC methods are used to regulate the MMC during AC unbalanced faults. Although they elaborate a comprehensive control method for the AC grid current, the internal energy balancing is disregarded.

The different approaches presented above share similar AC grid current control methods which only considers the injection of positive-sequence active current component even during AC network voltage sags. Under these faults, the MMC must comply with the TSO's Fault Ride-Through (FRT) requirements, providing additional support to the network (e.g. frequency or voltage supports), by either injecting and/or absorbing active and/or reactive current components. Although these requirements are imposed by the TSOs of each country, in accordance to the European Network of Transmission System Operators (ENTSO-E) [21], there is a limited number of publications addressing this issue in HVDC systems. In [22], a voltage support control strategy is introduced, but it only deals with balanced AC network fault scenarios. Unbalanced scenarios are analyzed in [23], whereby the control strategy consisted in disabling the positive active and reactive current components to maximize the negative part. Reference [24] considered both positive and negative components during the fault. Still, the prioritization proposed by [24] makes the MMC to exceed its AC grid current limits during transients. Authors in [25] introduced an optimization-based current reference calculation to compute the optimal references of the converter considering its design limitations. However, it performs the prioritization in the $a b c$ reference frame, which may be more challenging to impose the grid-code requirements (given based on symmetrical components). It also disregards the power transfer with the arms and phase-legs of the converter and requires high computational burden.

To the best of the authors knowledge, a real-time optimization-based reference calculation algorithm integrated with a control for MMC-HVDC stations to provide adequate support to a faulted network while considering the converter limitations has not been proposed yet. Aiming to address these challenges, this manuscript brings the following main contributions:

- Formulation of an optimization-based control strategy to ensure that the positive- and negative-sequences of the AC grid's active and reactive components are as close as possible to the grid code requirements, without exceeding the MMC's limits.

- The MMC's DC and AC circulating current components are limited simultaneously for each arm of the converter.

- The power mismatches between the converter's upper and lower arms, as well as among the phase-legs are considered simultaneously in the formulation of the algorithm.

- The computational burden of the algorithm is reduced by performing an LTV approximation of the system model. It is shown that this approach reduces the convergence time more than one order of magnitude compared to a nonlinear formulation; making the real-time implementation of the algorithm feasible.

- Definition of an strategy to achieve a seamless transition between the optimized and a conventional reference generator in the event that the optimization does not converge in the allowed time.

The proposed linearized optimization-based reference calculation algorithm integrated with the MMC's control strategy is analyzed theoretically and through detailed time-domain simulations. Section II describes the optimization problem, Section III performs a qualitative comparison between the nonlinear model and its linear equivalent (described in Section II-C). The transition between the optimized and a conventional reference calculation for cases where the optimization algorithm cannot find a solution within the specified time is discussed in Section IV. In Section V different cases studies are analyzed in order to validate the performance of the proposed linearized optimization algorithm for real-time applications. Section VI compares the proposed method with conventional MMC control strategies. Finally, the conclusions are drawn in Section VII.

\section{DESCRIPTION OF THE OPTIMIZATION PROBLEM}

This section describes the formulation of the optimization problem and the simplification applied to reduce its computational burden for real-time applications. It is first shown that the analysis of the MMC system leads to set of nonlinear equations. Thus, the preliminary nonlinear formulation of the optimization of the system is presented. Next, the LTV approximation is employed into the nonlinear model, which permits to formulate the optimization as a standard linear quadratic problem $(\mathrm{QP})$ reducing the computational complexity. The schematic of the MMC is shown in the left-part of Fig. 1, in which $u_{u, l}^{k}$ and $i_{u, l}^{k}$ represents the arms' voltages and currents, $u_{g}^{k}$ and $i_{s}^{k}$ are the AC network voltages while $U_{u, l}^{D C}$ are the HVDC link voltages, respectively. The arm and equivalent network impedances (used during the modelling derivation) are given as $R_{a}, L_{a}$ and $R_{s}, L_{s}$, respectively.

\section{A. Nonlinear model of the MMC}

For the nonlinear model, the phasor notation $\underline{X}^{k}=$ $X_{r}^{k}+j X_{i}^{k}=X^{k} \angle \theta^{k}$ will be adopted, with $\overline{x(t)}=$ $X^{k} \operatorname{Re}\left\{e^{j\left(\omega t+\theta^{k}\right)}\right\} \forall k \in(a, b, c)$. The steady-state mathematical equations of the MMC can be described as

$$
\begin{gathered}
\underline{U}_{0 n}=\underline{U}_{g}^{k}+\underline{Z}_{s}\left(\underline{I}_{u}^{k}-\underline{I}_{l}^{k}\right)+\underline{Z}_{a} \underline{I}_{u}^{k}+\underline{U}_{u}^{k} \\
\underline{U}_{0 n}=\underline{U}_{g}^{k}+\underline{Z}_{s}\left(\underline{I}_{u}^{k}-\underline{I}_{l}^{k}\right)-\underline{Z}_{a} \underline{I}_{l}^{k}-\underline{U}_{l}^{k} \\
\underline{I}_{u}^{a}+\underline{I}_{u}^{b}+\underline{I}_{u}^{c}=0 \\
\underline{I}_{s}^{a}+\underline{I}_{s}^{b}+\underline{I}_{s}^{c}=0 \\
\underline{I}_{s}^{k}=\underline{I}_{u}^{k}-\underline{I}_{l}^{k} \\
{\left[\begin{array}{l}
I_{s_{r}}^{+,-} \\
I_{s_{i}}^{+,-}
\end{array}\right]=\left[\begin{array}{ll}
\cos \left(\theta^{+,-}\right) & -\sin \left(\theta^{+,-}\right) \\
\sin \left(\theta^{+,-}\right) & \cos \left(\theta^{+,-}\right)
\end{array}\right] \cdot\left[\begin{array}{l}
\alpha^{+,-} \cdot I_{P}^{+,-} \\
\beta^{+,-} \cdot I_{Q}^{+,-}
\end{array}\right]} \\
{\left[\begin{array}{l}
\underline{I}_{s}^{a} \\
\underline{I}_{s}^{b} \\
\underline{I}_{s}^{c}
\end{array}\right]=\left[\begin{array}{ccc}
1 & 1 & 1 \\
\underline{p}^{2} & \underline{p} & 1 \\
\underline{p} & \underline{p}^{2} & 1
\end{array}\right] \cdot\left[\begin{array}{l}
I_{s_{r}}^{+}+j \cdot I_{s_{i}}^{+} \\
I_{s_{r}}^{-}+j \cdot I_{s_{i}}^{-}
\end{array}\right]}
\end{gathered}
$$




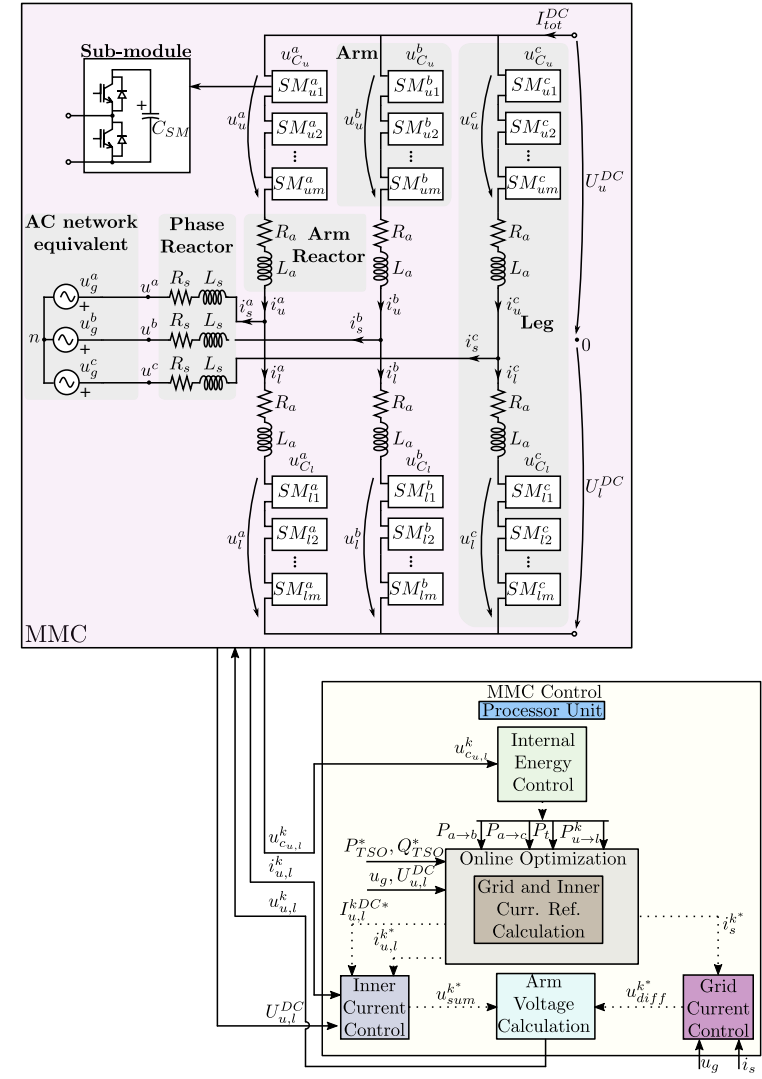

Fig. 1. MMC model with the proposed optimal control conceptualization.

$$
\begin{gathered}
\sum_{k=a}^{c}\left[\operatorname{Re}\left\{\underline{U}_{u}^{k} \underline{I}_{u}^{k}+\underline{U}_{l}^{k} \underline{I}_{l}^{k}\right\}+U_{u}^{k D C} I_{u}^{k D C}+U_{l}^{k D C} I_{l}^{k D C}\right]=0 \\
P_{u \rightarrow l}^{k}=\operatorname{Re}\left\{\underline{U}_{u}^{k} \underline{I}_{u}^{k}-\underline{U}_{l}^{k} \underline{I}_{l}^{k}\right\}+\left(U_{u}^{k D C} I_{u}^{k D C}-U_{l}^{k D C} I_{l}^{k D C}\right) \\
P_{a \rightarrow b}=\operatorname{Re}\left\{\left(\underline{U}_{u}^{a} \underline{I}_{u}^{a}+\underline{U}_{l}^{a} \underline{I}_{l}^{a}\right)-\left(\underline{U}_{u}^{b} \underline{I}_{u}^{b}+\underline{U}_{l}^{b} \underline{I}_{l}^{b}\right)\right\}+ \\
\left(U_{u}^{a D C} I_{u}^{a D C}+U_{l}^{a D C} I_{l}^{a D C}\right)-\left(U_{u}^{b D C} I_{u}^{b D C}+U_{l}^{b D C} I_{l}^{b D C}\right) \\
P_{a \rightarrow c}=\operatorname{Re}\left\{\left(\underline{U}_{u}^{a} \underline{I}_{u}^{a}+\underline{U}_{l}^{a} \underline{I}_{l}^{a}\right)-\left(\underline{U}_{u}^{c} \underline{I}_{u}^{c}+\underline{U}_{l}^{c} \underline{I}_{l}^{c}\right)\right\}+ \\
\left(U_{u}^{a D C} I_{u}^{a D C}+U_{l}^{a D C} I_{l}^{a D C}\right)-\left(U_{u}^{c D C} I_{u}^{c D C}+U_{l}^{c D C} I_{l}^{c D C}\right) \\
I_{s}^{k D C}=I_{u}^{k D C}-I_{l}^{k D C} \\
I_{t o t}^{D C}=I_{u}^{a D C}+I_{u}^{b D C}+I_{u}^{c D C} \\
U_{u}^{D C}+U_{l}^{D C}=U_{u}^{k D C}+U_{l}^{k D C}+2 R_{a}\left(I_{u}^{k D C}+I_{l}^{k D C}\right) \\
\left(-U_{u}^{a D C}+U_{l}^{a D C}\right)+\left(U_{u}^{b D C}-U_{l}^{b D C}+U_{u}^{c D C}-U_{l}^{c D C}\right)=0 \\
\left(U_{u}^{b D C}-U_{l}^{b D C}-U_{u}^{c D C}+U_{l}^{c D C}\right)=0
\end{gathered}
$$

with $\underline{p}=e^{j \frac{2 \pi}{3}}, \theta^{+,-}$being the AC grid voltage positiveand negative-sequences phase-angles and $I_{p}^{+,-}$and $I_{q}^{+,-}$as the positive- and negative-sequence active and reactive current components set-points to be injected/absorbed by the AC network which are demanded by the TSO, respectively. Equations (1a-1e) and (11-1m) describe the AC and DC currents and the AC voltages in the converter, already included in the optimization problem in [25]. Expressions (1f-1g) are used to calculate the $\mathrm{AC}$ network current levels. Note that the coefficients $\alpha^{+,-}$and $\beta^{+,-}$are used to adequate the amount of active and reactive currents to be injected/absorbed by the AC network in order to avoid the converter to exceed its physical limitations. In addition, the values of these four coefficients have a direct effect on the AC grid current magnitudes and an indirect impact on other internal quantities of the MMC, which may cause them to exceed their limits. Thus, the selection of $\alpha^{+,-}$and $\beta^{+,-}$is performed by an optimization algorithm (see Section II-B). Next, (1h-1p) introduce terms not accounted in [25]. Where, (1h) describes the steady-state condition, (1i-1k) define the power differences between arms and phase-legs and (1n-1p) characterize the DC voltages in the converter while eliminating possible DC voltages into the AC grid.

\section{B. Nonlinear optimization problem}

The multi-objective nonlinear optimization problem that enables the prioritization among the positive- and negativesequence components of the active and reactive AC grid current and, at the same time, ensures that the MMC's quantities are kept within their design limits is given as follows

$$
\begin{array}{cc}
\max _{\substack{I_{u, l}^{k}, I_{u, l}^{k D C}, \underline{U}_{u, l}^{k}, U_{u, l}^{k D C}, \underline{U}_{n 0}, \alpha^{+,-}, \beta^{+,-}}} & \lambda_{I_{p}^{+}} \alpha^{+}+\lambda_{I_{q}^{+}} \beta^{+}+\lambda_{I_{p}^{-}} \alpha^{-}+\lambda_{I_{q}^{-}} \beta^{-} \\
\text {s.t. } & (1 a \text { to } 1 p), \\
& I_{s}^{k} \leqslant I_{\max }^{A C}, \\
I_{u, l}^{k}+I_{u, l}^{k D C} \leqslant I_{\max }^{\text {arm }}, \\
0 \leqslant U_{u, l}^{k}+U_{u, l}^{k D C} \\
0 \leqslant \alpha^{+} \leqslant 1, \\
0 \leqslant \alpha^{-} \leqslant 1 \\
0 \leqslant \beta^{+} \leqslant 1 \\
0 \leqslant \beta^{-} \leqslant 1
\end{array}
$$

where, the OF, given in (2a), consists of four coefficients, $\alpha^{+,-}$and $\beta^{+,-}$, which are employed to regulate the levels of positive- and negative-sequence active and reactive current components to be injected/absorbed by the AC grid, respectively, in order to comply with the grid operator requirements. These coefficients are be prioritized according to the values used in the optimal weights $\lambda_{I_{p}+,-, I_{q}+,-}$. The optimal weights in this paper have been chosen based on the performance of the reactive current injection/absorption after running extensively simulations considering different faults and several different weights' combination. It was observed after those tests that the optimal performance that eases the prioritization and improve the convergence time it is when factor of at least $10^{-3}$, regarding each $\lambda_{x}$ is used. Then, in order to provide optimal voltage support to the network during AC network faults, the optimal weights are selected as $\lambda_{I_{q}^{+}} \gg \lambda_{I_{q}^{-}} \gg \lambda_{I_{p}^{+}} \gg \lambda_{I_{p}^{-}}{ }^{1}$. The equality constraints are set in (2b) and are based on

\footnotetext{
${ }^{1}$ Under unbalanced faults which require the injection of the positivesequence and the absorption of the negative-sequence reactive components, the positive-sequence current to be injected will be $90^{\circ}$ leading the phaseangle of $U_{g}^{+}$, whereas the current to be absorbed for $U_{g}^{-}$must be lagging $90^{\circ}$ in order to mitigate its asymmetric effects. For grid operators that prioritize the active current injection (e.g. [26]), the relation should be modified as $\lambda_{q}^{+,-} \ll \lambda_{p}^{+,-}$.
} 
the nonlinear steady-state model of the MMC (see Section II-A). The maximum allowed current in the $\mathrm{AC}$ network and through the MMC's arms are imposed in (2c-2d) by setting the magnitudes of $I_{\max }^{A C}$ and $I_{\max }^{a r m}$, respectively. Since halfbridge SMs are considered in this analysis, the lower limit in (2e) is set as 0 , as it is not possible to synthesize negative voltages. Nevertheless, negative values could be considered if full-bridge SMs were employed. Constraints (2f-2i) establish the maximum and minimum allowed values for the optimal weights $\alpha^{+,-}$and $\beta^{+,-}$. Under normal conditions, these four quantities are equal to 1 , but depending on the $\mathrm{AC}$ and DC network voltage conditions, their levels might change in order to meet the MMC limitations. This manuscript takes as study case the specifications of the Spanish TSO [27], whose requirements are to maximize the injection of reactive current during AC grid voltage sags. The magnitude of the additional reactive current component $\Delta I_{r}$ to be injected/absorbed by the grid varies according to the RMS voltage levels at the PCC as

$$
\begin{gathered}
U_{\min 1} \leqslant U_{g} \leqslant U_{\max 1} \rightarrow \Delta I_{r}=0 \\
U_{\min 2} \leqslant U_{g}<U_{\min 1} \rightarrow \Delta I_{r}=\frac{\Delta I_{r \max }\left(U_{\min 1}-U_{g}\right)}{U_{\min 1}-U_{\min 2}} \\
U_{g}<U_{\min 2} \rightarrow \Delta I_{r}=\Delta I_{r \max }
\end{gathered}
$$

where the values of $U_{\min 1}, U_{\max 1}, U_{\min 2}$ and $\Delta I_{r \max }$ are defined by the grid operator.

\section{Linearization of the optimization problem}

Although most of the equations presented in Section II-B are linear, the internal energy balance of the converter (1i-1k) and the overall energy balance of the system (1h) present a strong nonlinear relationship. This problem is similar in nature to the energy regulation problem discussed in [28] where an LTV approximation is proposed to reduce the computational burden of a nonlinear MPC controller. This same linearization strategy is implemented in [29] to design a predictive controller on an MMC controller; thus validating the scalability of the LTV approach to the MMC. Similarly, the LTV linearization is adopted here to tackle the large computational burden arisen from the nonlinear formulation of the prioritization algorithm.

Therefore, the nonlinear behaviour of the system is decomposed into two different subsystems: one that determines the nonlinear behaviour of the converter and is characterized by its steady-state trajectories, and an LTI system which describes the small-signal perturbations around the nonlinear steadystate points. This approximation resembles a classic Jacobian linearization. However, in this case, it is performed over steady-state varying trajectories instead of static DC quiescent point. Note that these steady-state trajectories can either be calculated within the optimization algorithm or come from an external source (see Section IV). Generically, the LTV approximation can be represented as

$$
\begin{aligned}
\frac{d}{d t} x & =\frac{d}{d t}(X+\hat{x})=\frac{d}{d t} X+\frac{d}{d t} \hat{x}=f(x, u) \\
& \approx f(X, U)+\left.\frac{\partial f}{\partial x}\right|_{\hat{x}, \hat{u}}(x-X)+\left.\frac{\partial f}{\partial u}\right|_{\hat{x}, \hat{u}}(u-U) \\
& \approx \underbrace{f(X, U)}_{F_{0}}+\frac{\partial f(X, U)}{\partial x} \hat{x}+\frac{\partial f(X, U)}{\partial u} \hat{u}
\end{aligned}
$$

where $x$ represents a vector containing the MMC's states $x \triangleq$ $\left[\underline{I}_{u_{[1]}}^{k}, \underline{I}_{l_{[1]}}^{k}, I_{u_{[1]}}^{k D C}, I_{l_{[1]}}^{k D C}, \underline{I}_{s_{[1]}}^{k}, \alpha_{[1]}^{+}, \beta_{[1]}^{+}, \alpha_{[1]}^{-}, \beta_{[1]}^{-}, \ldots, \underline{I}_{u_{[N]}}^{k}\right.$, $\left.\underline{I}_{l_{[N]}}^{k}, I_{u_{[N]}}^{k D C}, I_{l_{[N]}}^{k D C}, \underline{I}_{s_{[N]}}^{k}\right]^{T}, \quad u \quad$ accounts for the converter's control quantities $u \triangleq\left[\underline{U}_{u_{[0]}}^{k}, \underline{U}_{l_{[0]}}^{k}, U_{u_{[0]}}^{k D C}\right.$, $U_{l_{[0]}}^{k D C}, \ldots, \underline{U}_{u_{[N-1]}}^{k}, \underline{U}_{l_{[N-1]}^{k}}^{k}, U_{u_{[N-1]}}^{k D C}, U_{l_{[N-1]}^{k D C}}^{k D} \quad$ and $\quad \mathbf{F}_{0}$ represents the steady-state trajectories (see Section IV).

1) Linearized $O F$ and equality constraints: The $\mathrm{OF}$ and equality constraints of the linearized optimiziation problem are obtained by applying (4) in the expressions given in $(2 \mathrm{a}-2 \mathrm{~b})$.

2) Linearized inequality constraints: The inequalities relating the optimal weights $\alpha^{+,-}$and $\beta^{+,-}$already have a linear profile; thus, applying (4) into $(2 \mathrm{f}-2 \mathrm{i})$ results in

$$
\begin{aligned}
& 0 \leqslant\left(A^{+}+\hat{\alpha}^{+}\right) \leqslant 1, \quad 0 \leqslant\left(A^{-}+\hat{\alpha}^{-}\right) \leqslant 1, \\
& 0 \leqslant\left(B^{+}+\hat{\beta}^{+}\right) \leqslant 1, \quad 0 \leqslant\left(B^{-}+\hat{\beta}^{-}\right) \leqslant 1
\end{aligned}
$$

Unlikely the previous inequalities, the current and voltage inequality constraints in (2c-2e) have nonlinear circular characteristics, as they are calculated based on absolute values relating the real and imaginary parts of the current phasors. One possible way to linearize the aforementioned inequality constraints is to employ (4). But neglecting the high-order nonlinear terms may lead the MMC to exceed its current limitations or impose negative voltages into the arms (which is not possible considering that half-bridge structures are employed into the SMs).

In order to overcome the aforementioned drawbacks introduced by the linearization of the absolute value constraints, a potential solution is proposed based on the decomposition of these constraints into two complementary components in quadrature. Then, the sum of these two signals, modelled as sinusoidal trajectories, must always be within the maximum and minimum values imposed by the converter limitations (see $(2 \mathrm{c}-2 \mathrm{e})$ ). Note that the accuracy of this approximation is now limited by the number of points $N_{\text {points }}$ considered to represent these quadrature components (e.g. higher number of points results in higher accuracy; but, higher computational burden). The sine waves are mathematically described in (6) and represented (for different time instants) in Fig. 2.

$$
\begin{gathered}
\operatorname{Re}=\left(X_{r}+\hat{x_{r}}\right) \cos \left(\omega n_{k} T_{s}\right), \quad \operatorname{Im}=\left(X_{i}+\hat{x_{i}}\right) \sin \left(\omega n_{k} T_{s}\right) \\
\operatorname{Mag}=\left(X_{r}+\hat{x_{r}}\right) \cos \left(\omega n_{k} T_{s}\right)+\left(X_{i}+\hat{x_{i}}\right) \sin \left(\omega n_{k} T_{s}\right)
\end{gathered}
$$

where $\omega=100 \pi$.

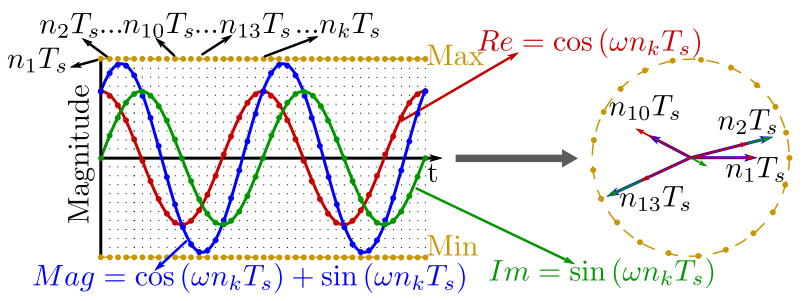

Fig. 2. Linearization for AC grid currents and arms' current and minimum applied voltage inequalities.

Therefore, applying (6) into the current inequalities in (2a), the linearized constrains to limit the AC grid and arm's 
currents and the minimum voltage to be applied to the arm's can be obtained and are expressed as

$$
\begin{aligned}
& -I_{\text {max }}^{A C} \leqslant\left(I_{s_{r}}^{k}+\hat{i}_{s_{r}}^{k}\right) \cos \left(\omega n_{k} T_{s}\right)+\left(I_{s_{i}}^{k}+\hat{i}_{s_{i}}^{k}\right) \sin \left(\omega n_{k} T_{s}\right) \leqslant I_{\text {max }}^{A C} \text { (7a) } \\
& -I_{\text {max }}^{a r m} \leqslant\left(I_{u, l_{r}}^{k}+\hat{i}_{u, l_{r}}^{k}\right) \cos \left(\omega n_{k} T_{s}\right)+\left(I_{u, l_{i}}^{k}+\hat{i}_{u, l_{i}}^{k}\right) \sin \left(\omega n_{k} T_{s}\right)+ \\
& \left(I_{u, l}^{k D C}+\hat{i}_{u, l}^{k D C}\right) \leqslant I_{m a x}^{a C m} \\
& 0 \leqslant\left(U_{u, l_{r}}^{k}+\hat{u}_{u, l_{r}}^{k}\right) \cos \left(\omega n_{k} T_{s}\right)+\left(U_{u, l_{i}}^{k}+\hat{u}_{u, l_{i}}^{k}\right) \sin \left(\omega n_{k} T_{s}\right)+\quad \text { (7b) } \\
& \quad\left(U_{u, l}^{k D C}+\hat{u}_{u, l}^{k D C}\right)
\end{aligned}
$$

\section{ANALYSIS OF THE LINEARIZED OPTIMIZATION PROBLEM}

In this section, the presented linearized optimization algorithm is compared with a nonlinear optimization algorithm tuned with similar tolerance levels as the linear approximation, and a nonlinear algorithm tuned with very fine tolerance (case considered as the reference solution). The comparative analysis is performed in both normal and faulted AC grid voltage scenarios. The nonlinear problem is obtained using Matlab ${ }^{\circledR}$ fmincon function (Sequential Quadratic Program (SQP) based algorithm [30]) and the Operator Splitting Quadratic Program (OSQP) solver [31] is used to find the solution of the equivalent linearized problem. The comparison relies on two main aspects - accuracy (which relates to the OF and optimal weights' $\alpha^{+,-}$and $\beta^{+,-}$values) and convergence time $T_{\text {conv }}$, considering that different number of points are employed in the sinusoidal waveforms to calculate the inequality constraints (see Section II-C2). Based on these parameters, $N_{\text {points }}$ is selected to be later used in the online optimization algorithm in order to calculate the MMC's current references (see Section V).

\section{A. Case 1 - Normal AC grid conditions}

In this case study, the MMC is considered to be operated under an unconstrained scenario with normal AC grid voltage conditions. In the upper-left part of Fig. 3, the value of the $\mathrm{OF}$ is depicted. As it can be noted, both nonlinear and linear (regardless the number of points employed in the inequality constraints) models result in similar magnitude as the reference one. Regarding the convergence time, the solution of the nonlinear model is obtained in approximately $90 \mathrm{~ms}$; in contrast, the linearized problem is solved within $2.5 \mathrm{~ms}$ (nearly 36 times faster, assuming $N_{\text {points }}>5$ ). For the optimal weights, $\alpha_{\text {ref }}^{+}=\alpha_{\text {lin }}^{+}=1$ while its nonlinear value has a small deviation being equal to $\alpha_{\text {non }}^{+}=0.98$. Whereas for $\beta^{+,-}$, both nonlinear and linear optimization algorithms output the same number as the desired one $\beta_{r e f}^{+,-}=1$. Finally, for this $\mathrm{AC}$ network condition, any number of points above 5 could be selected to be employed in the online algorithm.

\section{B. Case 2 - Balanced AC grid fault}

For this scenario, an analogous analysis is performed assuming that the $\mathrm{AC}$ network is under a balanced three-phase voltage sag. The $\mathrm{OF}$ value for both the nonlinear model and the linear optimization are in close-agreement with the desired one, as shown in Fig. 4. In addition, the convergence time is greatly improved by using the linearized model (around 35 times faster assuming $N_{\text {points }} \geqslant 6$ ). Regarding the optimal
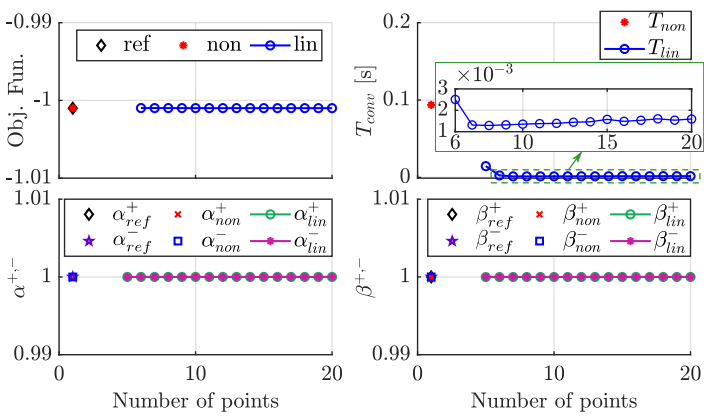

Fig. 3. Comparison between the nonlinear and linear models under normal $\mathrm{AC}$ grid operations and with relative and absolute tolerances equal to $10^{-3}$.

weights, both $\alpha_{\text {non }}^{+,-}$and $\alpha_{\text {lin }}^{+,-}$have similar values to the very fine tolerance optimization problem. For $\beta$, the nonlinear ones are equal to the reference magnitude, whereas $\beta_{\text {lin }}^{+}$have fluctuations around the desired point (respective to the number of points employed in the inequality constraints). Based on the analysis of the previous parameters, under this type of fault $N_{\text {points }}=7$ would be an optimal number of points to be employed in the inequality constraints.
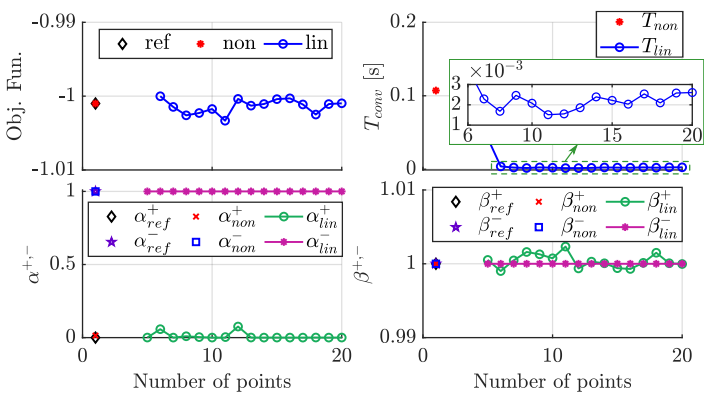

Fig. 4. Comparison between the nonlinear and linear optimization under balanced AC three-phase voltage sag.

\section{Case 3 - Unbalanced AC grid faults}

The performance of the linearized model is analyzed considering an unbalanced $\mathrm{AC}$ voltage sag types $\mathrm{C}$ [32]. Observing Fig. 5a, it is clear that the linearized optimal weights present a disparity from their respective reference values, especially for $\alpha_{l i n}^{+}$and $\beta_{l i n}^{+}$, regardless the number of points employed in the inequality constraints. Such mismatches can be reduced if the solver tolerances are tightened, as it shown in Fig. $5 \mathrm{~b}$. For the first case, the absolute and relative tolerances are set to be equal to $10^{-3}$, whereas in the second one, the tolerances are reduced to $10^{-6}$. Although the precision of the solver is improved, it is important to highlight that there is a trade off between the convergence time and the solver's precision. As it can be noted in Fig. 5, by tightening the solver's tolerance, the convergence time $T_{\text {conv }}$ for the linearized model is increased reaching infeasible values to be used in the proposed optimization algorithm to calculate the MMC's current reference in online applications.

Finally, due to the responses of the linearized optimized model for balanced and unbalanced AC grid scenarios and the precision and convergence time of the solver, the number of points to be used in the online optimization algorithm is equal 
to $N_{\text {points }}=7$ and the absolute and relative tolerances are set to $10^{-3}$

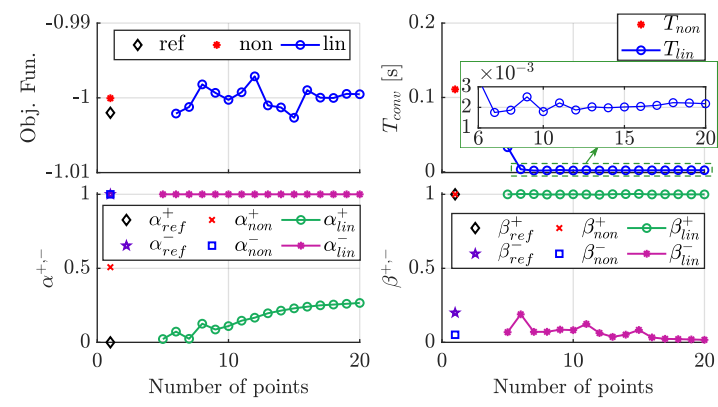

(a) Absolute and relative tolerances equal to $10^{-3}$.

Still considering the same unbalanced voltage scenario, number of points and formulation of the linearized optimization problem, the time-to-solution of the algorithm is depicted in Fig. 6 considering different solvers. In this Figure, only the solution time of the optimization algorithm is considered once all the matrices involved in the problem have been formulated; thus, the convergence time is smaller compared to the previous cases. It is clear that not only the selection of the former discussed parameters (e.g. tolerances, number of points) have an important impact on the convergence time of the optimization problem, but also the solver employed. Finally, the OSQP solver presented better performance among the different solvers, reducing the convergence time by more than one order of magnitude.

\section{INTEGRATION WITH THE CONTROLLER}

In this section, the optimization-based reference calculation algorithm is integrated with the overall control structure of the converter. The control scheme implemented is shown in Fig. 7 and uses the design procedures derived in [15]. This control strategy tracks the $\mathrm{AC}$ and $\mathrm{DC}$ current components in the stationary $\alpha \beta 0$ reference frame and can be separated into three main parts: the $\mathrm{AC}$ grid and circulating currents controls and the internal energy regulators. The energy controllers are required to keep the internal energy of the converter balanced. This is achieved by regulating the total energy of the converter $E_{t}$, the energy differences between the upper and lower arms $E_{u \rightarrow l}^{k}$ and among the MMC's phase-legs $E_{a \rightarrow b}$ and $E_{a \rightarrow c}$. These energy controls output the power set-points to calculate

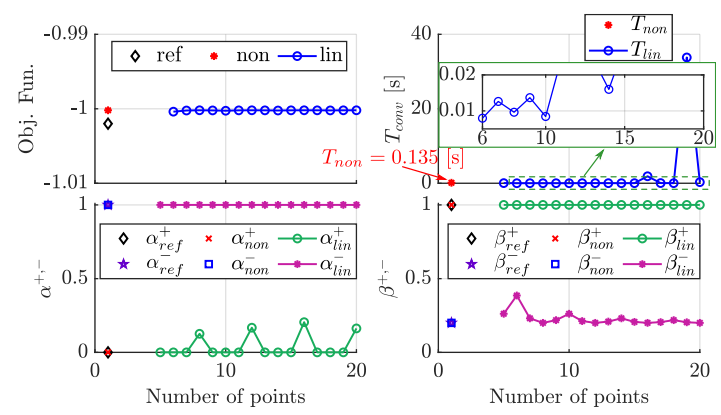

(b) Absolute and relative tolerances equal to $10^{-6}$.

Fig. 5. Comparison between the nonlinear and linear optimization for a type C fault.

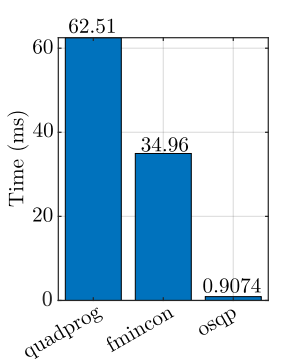

Fig. 6. Computation time required by different solvers. The comparison is performed running the algorithm on an Intel i7-4770 CPU @ $3.40 \mathrm{GHz}$.

the non-optimal $\mathrm{AC}$ and $\mathrm{DC}$ circulating currents references. For the AC network currents, the non-optimal references are calculated based on the positive- and negative-sequence active and reactive current components demanded by the TSO and the magnitude of the positive- and negative-sequence components of the AC grid voltages. Besides the aforementioned nonoptimal reference calculations, the online optimization-based reference calculation block is also added as part of the overall control scheme (highlighted in yellow color in Fig. 7). The selection between the optimal and the non-optimal references is performed by the enable block (blue color block in Fig. 7) whose working principle is described later.

\section{A. AC network current reference}

The AC grid currents may present symmetrical or asymmetrical profiles under balanced and unbalanced conditions, respectively. This asymmetry is caused due to the presence of negative-sequence components, which must be taken into account during the reference calculation stage. As it can be noted in the upper part of Fig. 7, for both conventional and optimal calculation approaches, the positive- and negativesequence components of the active and reactive currents are pre-calculated based on [33]. Now, these values will be used for both online and conventional reference calculation. For the online method, such quantities are directly used as input parameters for the optimization algorithm. On the other hand, for the conventional method, the active and reactive current components are first saturated in order to ensure that their magnitudes are within the design limitations of the converter. Then, the saturated currents are employed in the grid side reference calculation block, resulting in the traditional AC network current references $i_{s_{c o n v}}^{\alpha \beta 0^{*}}$ which will also be used as input parameters for the online calculation strategy as the steady-state trajectories required by the linearized model. Finally, depending on the output value of enable block, either the optimal $i_{s_{o p t}}^{\alpha \beta 0^{*}}$ or the conventional $i_{s_{c o n v}}^{\alpha \beta 0^{*}}$ current references are sent to the grid side current control.

\section{B. Additive current reference}

The MMC's circulating currents contain both AC and DC components, which are employed to regulate the internal energy balance of the converter. Such inner current set-points are first calculated through the method described in [34] without considering the limitations of the converter. Then, these values are used as the steady-state trajectories of the online optimization algorithm. Although the trajectory levels 


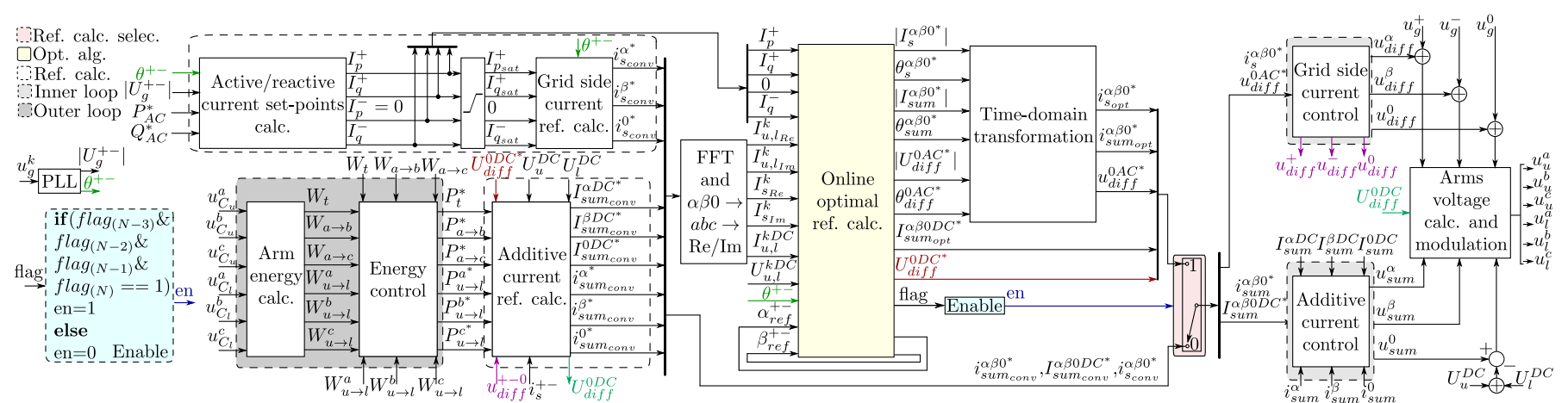

Fig. 7. Full control scheme of the MMC integrating the optimal reference calculation.

may be exceeding the MMC's internal current limits, the optimization problem is derived in a manner that allows its small-signal values to adjust their magnitudes. Therefore, the final current value output by the optimization algorithm will be kept within the physical limitations of the converter (see Section II-C2).

According to the enable block's output, the additive current control block may either use the adequate saturated and optimized inner current $i_{s u m_{o p t}}^{\alpha \beta 0}$ or the non-optimal values $i_{\text {sum }}^{\alpha \beta 0}{ }_{\text {conv }}$ as the current references required by the control strategy. In which, for the non-optimal values, hard saturation strategies are employed for both DC and AC $\alpha \beta 0$ additive current components. However, such approach cannot ensure the optimal usage of the converter.

\section{Backup reference calculation method}

The enable block is responsible for integrating the optimization algorithm with the MMC's controllers. Its main objective is to ensure that the control scheme is maintained properly running even in a hypothetical scenario where the algorithm can not converge into a solution within the control time step. The desired output flag level is equal to 1 , meaning that the optimization algorithm is able to converge into a solution that satisfies the problem constraints (see Section II-C). The enable scheme keeps measuring the optimization flag and, if the flags are kept equal to 1 during the consecutive sample $T_{N} \rightarrow T_{N-3}$, used as a safety factor, then the optimal references are fed into the controllers. However, if at the time instant $T_{N}$ the output flag is different than 1, the non-optimized references are imposed into the controllers.

While the optimal reference generator is over-ruled, the optimization problem keeps being solved in the background. If in the next time steps the algorithm is capable of solving the problem within the specified time, the enable block returns to feed the controller with the optimized references. Otherwise, the conventional non-optimized reference are kept used until the optimization algorithm is able to converge satisfactorily; thus guaranteeing the safe operation of the system.

\section{REsults}

This section presents the results obtained when the proposed optimization-based control is employed to regulate the MMC under different operating conditions. For the first case study, the amount of reactive and active currents injected into the
AC grid during distinct voltage sags is compared. Cases II to IV present the time-domain waveforms of the MMC when it is operated under balanced and unbalanced AC and DC network voltage conditions. Finally, the performance of the optimization algorithm is analyzed based on its convergence time for the different $\mathrm{AC}$ grid voltage sags and DC unbalances. Even though in real networks the maximum allowed time for fault-ride through is $250 \mathrm{~ms}$ [35], the fault events under study are extended to $500 \mathrm{~ms}$ to validate the control scheme stability. The simulations are conducted on a computer with a 3.4 GHz Intel core i7-4770 processor with 16 GB of RAM using Matlab Simulink ${ }^{\circledR}$ considering an accelerated model of the MMC [36] and employing the Nearest Level Control (NLC) technique to calculate the number of active sub-modules in each arm [37]. The linear optimization problem is solved by the Operator Splitting Quadratic Program (OSQP) [31]. The time-domain waveforms are analyzed for the former conditions in order to confirm the applicability of the suggested method in real-time applications based on its performance for different requirements (e.g. convergence time and compliance with the MMC constraints). The system parameters employed for the case studies are detailed in Table I.

TABLE I

SYSTEM PARAMETERS

\begin{tabular}{|c|c|c|c|}
\hline Parameter & Symbol & Value & Units \\
\hline Rated power & $S$ & 1000 & MVA \\
\hline Rated power factor & $\cos \phi$ & $0.95(\mathrm{c})$ & \\
\hline AC-side rated voltage & $\underline{U}_{q}$ & 325 & $\mathrm{kV}$ \\
\hline HVDC link voltage & $\overrightarrow{U^{D C} C}$ & \pm 320 & $\mathrm{kV}$ \\
\hline Phase reactor impedance & $\underline{Z}_{s}$ & $0.005+\mathrm{j} 0.18$ & pu \\
\hline Arm reactor impedance & $\underline{\bar{Z}}_{a}^{s}$ & $0.01+\mathrm{j} 0.15$ & pu \\
\hline Converter modules per arm & $N_{u, l o v}^{k^{-u}}$ & 433 & - \\
\hline Sub-module capacitance & $C_{S M}$ & 9.5 & $\mathrm{mF}$ \\
\hline Sample time & $T_{s}$ & 10 & $\mu \mathrm{s}$ \\
\hline Optimization time step & $T_{\text {step }}$ & 2.5 & $\mathrm{~ms}$ \\
\hline$I_{q}^{+,-}$injection response time & $t_{1}$ & 30 & $\mathrm{~ms}$ \\
\hline$I_{q}^{+},-$clearance response time & $t_{2}$ & 50 & $\mathrm{~ms}$ \\
\hline Maximum reactive current & $I_{a m}^{+,-}$ & $\sqrt{2}$ & $\mathrm{pu}$ \\
\hline Optimal weighting factor 1 & $\lambda_{I_{+}^{+}}$ & $10^{-6}$ & - \\
\hline Optimal weighting factor 2 & $\lambda_{I_{q}^{+}}^{p}$ & 1 & - \\
\hline Optimal weighting factor 3 & $\lambda_{I_{p}{ }^{-}}$ & $10^{-9}$ & - \\
\hline Optimal weighting factor 4 & $\lambda_{I^{-}}{ }^{p}$ & $10^{-3}$ & - \\
\hline Maximum MMC arm current & $I_{m a x}^{a r m}$ & 1.0842 & $\mathrm{pu}$ \\
\hline Maximum AC grid current & $I_{\max }^{A A C}$ & $\sqrt{2}$ & pu \\
\hline
\end{tabular}

\section{A. Case study I: AC grid current comparison}

In this case study, the steady-state values obtained using the proposed optimization-based current reference calculation 
algorithm considering different prioritizations are compared with the grid code demands. The TSO's requirements do not take into account the limitations of the converter, focusing exclusively in the unsaturated injection of positive- and negativesequence active and reactive current components (refereed as TSO's requirements). In the first prioritization scenario, named Proposal I, the proposed optimization method prioritizes the positive-sequence reactive and active components over the negative ones by employing optimal weights for the positivesequence quantities higher than the negative ones as $\lambda_{I_{q}^{+}}=1$, $\lambda_{I_{p}^{+}}=10^{-3}, \lambda_{I_{q}^{-}}=10^{-6}$ and $\lambda_{I_{p}^{-}}=10^{-9}$. Next, in Proposal II, the values of the optimal weights are changed to $\lambda_{I_{q}^{+}}=1, \lambda_{I_{q}^{-}}=10^{-3}, \lambda_{I_{p}^{+}}=10^{-6}$ and $\lambda_{I_{p}^{-}}=10^{-9}$ (as in Table I), prioritizing the reactive components over the active ones. Finally, the AC currents obtained from the previous schemes and the results gathered from the proposed linearized optimization are shown in Table II for several types of AC voltage sags considering $V=0.3 \mathrm{pu}$ [32].

As it can be observed in Table II, during the balanced fault scenario both Proposals I and II prioritize the injection of reactive current components over the active ones to provide full voltage support to the AC grid, as the converter has achieved its limitations. For the unbalanced AC grid faults type B to D, Proposal I can provide full positive-sequence voltage support to the grid and partial injection of positivesequence active current, as the MMC reaches its $\mathrm{AC}$ grid current limitations. On the other hand, as Proposal II prioritizes the negative-sequence reactive current component over the positive-sequence active one, it is capable of providing not only full positive-sequence voltage support during all faults, but also full (Fault type B) and partial (Faults C and D) negative-sequence voltage support. However, as the converter has reached its AC grid current limitations, Proposal II cannot inject active current into the grid. Finally, for Faults type E to G, both Proposals I and II have equal responses as the TSO's requirements for the positive-sequence reactive current component already imposes the converter to reach its limits.

\section{B. Case study II: Balanced AC voltage sag}

This case study is conducted to illustrate the performance of the full control scheme under a three-phase AC network voltage sag. The MMC is considered to be operating under normal conditions: $P=0.95 \mathrm{pu}, Q=0$ and $\left|U_{g}\right|^{k}=1 \mathrm{pu}$. Then, at $t=0.5 \mathrm{~s}$ a three-phase voltage sag is imposed into the AC system reducing the AC network voltage to $30 \%$ of its pre-fault value, and at $t=1 \mathrm{~s}$ the fault is cleared. Although the fault event happens in a abruptly manner, the injection of reactive current to provide voltage support to the grid must be done in agreement with the TSO's fault-ride through and magnitude requirements. According to the Spanish gridcode, if $\left|U_{g}\right|<0.6 \mathrm{pu}$, then the MMC must provide full voltage support to the system. The time-domain trajectories of the MMC states are displayed in Fig. 8, where the upper part shows the fault-ride through period and the bottom-half depicts the MMC's profile during the fault clearance course. In addition, it can be noted that both the $\mathrm{AC}$ and internal currents, as well as, the minimum allowed arms' applied voltages of the converter are respecting their limits (imposed during the formulation of the optimization problem) throughout the whole operation.

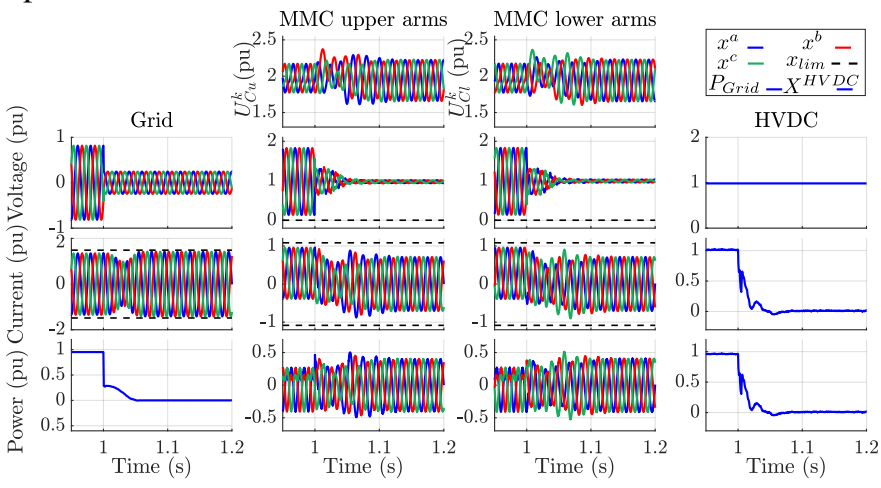

(a) Normal to fault.

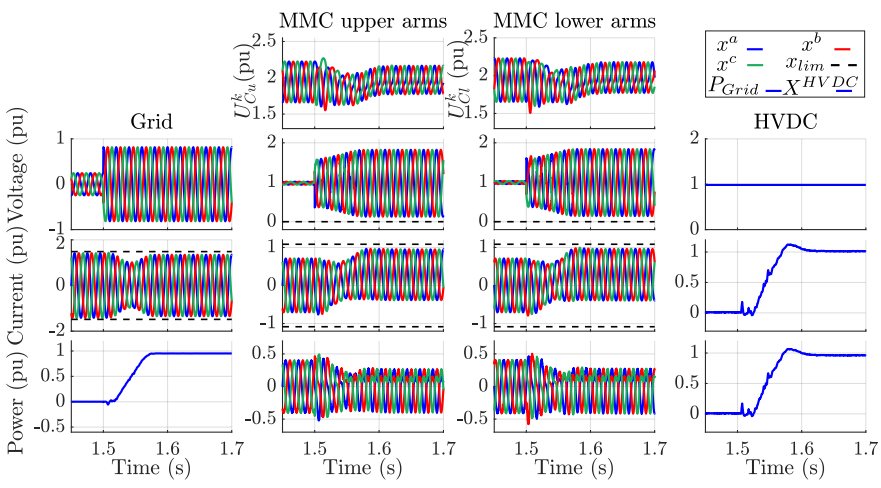

(b) Fault to normal.

Fig. 8. Time-domain waveforms for MMC's main quantities. a) From normal to fault scenario, b) From fault to normal operations.

The TSO also demands that the reactive current injection must be done within $30 \mathrm{~ms}$ after the fault event [27] and that the pre-fault active and reactive current set-points must be restored within $50 \mathrm{~ms}$ after the fault is cleared. In Fig. 9, the positive- and negative-sequence active and reactive current components profiles are shown. It can be noted that the optimal control scheme is able to provide additional reactive current to the faulted grid within the time range requested by the TSO. In addition, due to the balanced characteristics of the fault, the optimization method resulted in $I_{p_{o p t}}^{+,-}=0, I_{q_{o p t}}^{-}=0$ and $I_{q_{o p t}}^{+}=-1 \mathrm{pu}$ (full voltage support).

\section{Case study III: Unbalanced AC voltage sags}

Similarly to the previous scenario, this case study analyzes the online control scheme under an unbalanced voltage sag type $\mathrm{C}$, with $V=0.3 \mathrm{pu}$. The fault event and its clearance happen under same pre-fault conditions and times, as presented previously. Under this type fault, the grid voltages are equal to $\left|U_{g}^{+}\right|=0.65 \mathrm{pu}$ and $\left|U_{g}^{-}\right|=0.35 \mathrm{pu}$; thus, based on the Spanish grid-code, the MMC should provide partial positiveand negative-sequences reactive current components to the $\mathrm{AC}$ network. In Fig. 10, the MMC's waveforms are displayed and it can be observed that the converter's quantities are also kept within their limits. Differently from the previous case, during this fault condition there is a double-line oscillations in the 
TABLE II

COMPARISON OF THE STEADY-STATE ACTIVE AND REACTIVE CURRENTS CONSIDERING DIFFERENT PRIORITIZATIONS

\begin{tabular}{c|c|ccc|ccc}
\hline \hline \multirow{2}{*}{ Fault Type } & Voltage Level $[\mathrm{pu}]$ & \multicolumn{3}{|c}{$I_{p}^{+,-}[\mathrm{pu}]$} \\
& TSO's requirement & Proposal I & Proposal II & TSO's requirement & $\begin{array}{c}I_{q}^{+,-}[\mathrm{pu}] \\
\text { Proposal I }\end{array}$ & Proposal II \\
\hline \multirow{2}{*}{$\mathrm{A}$} & $U_{g}^{+}=0.30$ & $I_{p}^{+}=0.95$ & $I_{p}^{+}=0$ & $I_{p}^{+}=0$ & $I_{q}^{+}=-1$ & $I_{q}^{+}=-1$ & $I_{q}^{+}=-1$ \\
& $U_{g}^{-}=0$ & $I_{p}^{-}=0$ & $I_{p}^{-}=0$ & $I_{p}^{-}=0$ & $I_{q}^{-}=0$ & $I_{q}^{-}=0$ & $I_{q}^{-}=0$ \\
\hline \multirow{2}{*}{$\mathrm{B}$} & $U_{g}^{+}=0.767$ & $I_{p}^{+}=0.95$ & $I_{p}^{+}=0.896$ & $I_{p}^{+}=0.458$ & $I_{q}^{+}=-0.444$ & $I_{q}^{+}=-0.444$ & $I_{q}^{+}=-0.444$ \\
& $U_{g}^{-}=0.233$ & $I_{p}^{-}=0$ & $I_{p}^{-}=0$ & $I_{p}^{-}=0$ & $I_{q}^{-}=0.444$ & $I_{q}^{-}=0$ & $I_{q}^{-}=0.444$ \\
\hline \multirow{2}{*}{$\mathrm{C}$} & $U_{g}^{+}=0.65$ & $I_{p}^{+}=0.95$ & $I_{p}^{+}=0.553$ & $I_{p}^{+}=0$ & $I_{q}^{+}=-0.833$ & $I_{q}^{+}=-0.833$ & $I_{q}^{+}=-0.833$ \\
& $U_{g}^{-}=0.35$ & $I_{p}^{-}=0$ & $I_{p}^{-}=0$ & $I_{p}^{-}=0$ & $I_{q}^{-}=0.833$ & $I_{q}^{-}=0$ & $I_{q}^{-}=0.276$ \\
\hline \multirow{2}{*}{$\mathrm{D}$} & $U_{g}^{+}=0.65$ & $I_{p}^{+}=0.95$ & $I_{p}^{+}=0.553$ & $I_{p}^{+}=0$ & $I_{q}^{+}=-0.833$ & $I_{q}^{+}=-0.833$ & $I_{q}^{+}=0.833$ \\
& $U_{g}^{-}=0.35$ & $I_{p}^{-}=0$ & $I_{p}^{-}=0$ & $I_{p}^{-}=0$ & $I_{q}^{-}=0.833$ & $I_{q}^{-}=0$ & $I_{q}^{-}=0.167$ \\
\hline \multirow{2}{*}{$\mathrm{E}$} & $U_{g}^{+}=0.533$ & $I_{p}^{+}=0.95$ & $I_{p}^{+}=0$ & $I_{p}^{+}=0$ & $I_{q}^{+}=-1$ & $I_{q}^{+}=-1$ & $I_{q}^{+}=-1$ \\
& $U_{g}^{-}=0.233$ & $I_{p}^{-}=0$ & $I_{p}^{-}=0$ & $I_{p}^{-}=0$ & $I_{q}^{-}=0.444$ & $I_{q}^{-}=0$ & $I_{q}^{-}=0$ \\
\hline \multirow{2}{*}{$\mathrm{F}$} & $U_{g}^{+}=0.533$ & $I_{p}^{+}=0.95$ & $I_{p}^{+}=0$ & $I_{p}^{+}=0$ & $I_{q}^{+}=-1$ & $I_{q}^{+}=-1$ & $I_{q}^{+}=-1$ \\
& $U_{g}^{-}=0.233$ & $I_{p}^{-}=0$ & $I_{p}^{-}=0$ & $I_{p}^{-}=0$ & $I_{q}^{-}=0.444$ & $I_{q}^{-}=0$ & $I_{q}^{-}=0$ \\
\hline \multirow{2}{*}{$\mathrm{G}$} & $U_{g}^{+}=0.533$ & $I_{p}^{+}=0.95$ & $I_{p}^{+}=0$ & $I_{p}^{+}=0$ & $I_{q}^{+}=-1$ & $I_{q}^{+}=-1$ & $I_{q}^{+}=1$ \\
& $U_{g}^{-}=0.233$ & $I_{p}^{-}=0$ & $I_{p}^{-}=0$ & $I_{p}^{-}=0$ & $I_{q}^{-}=0.444$ & $I_{q}^{-}=0$ & $I_{q}^{-}=0$ \\
\hline \hline
\end{tabular}

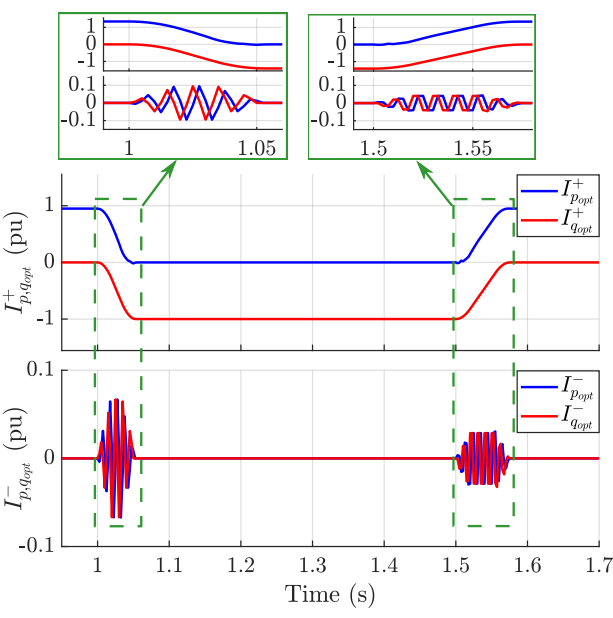

Fig. 9. $I_{p, q}^{+}$and $I_{p, q}^{-}$profiles during a three-phase fault.

AC grid power caused by the unbalanced characteristics of the fault. But, such fluctuations are not reflected to the DC side of the converter since the MMC energy storage cells can mitigate these effects.

In Fig. 11 the energy mismatches between the upper and lower arms, as well as, among the phase-legs of the converter are displayed. It can be observed that the proposed optimal control strategy is capable of compensating the energy deviations during the fault and the transitory profile is kept below $0.05 \mathrm{pu}$ (avoiding the converter protections to trip).

Now, the effects of the delays caused by the solving time of the optimization algorithm are analyzed during an SingleLine to Ground (SLG) fault scenario. It can be noted from Fig. 12 that during steady-state conditions, the delays have no effect as the waveforms considering the delays are in close agreement with the ideal ones. During transients, it is clear from Fig. 12a that the delayed upper arm currents present small deviations in contrast to the ideal trajectories. However, such deviations have negligible impact in the AC grid currents injected/absorbed by the converter during the fault as the ideal waveforms are matching the delayed ones (see Fig. 12b). In addition, all the other results presented in the paper indicate a better performance of proposed optimizationbased reference calculation integrated control in contrast to other control methods (see Section VI).
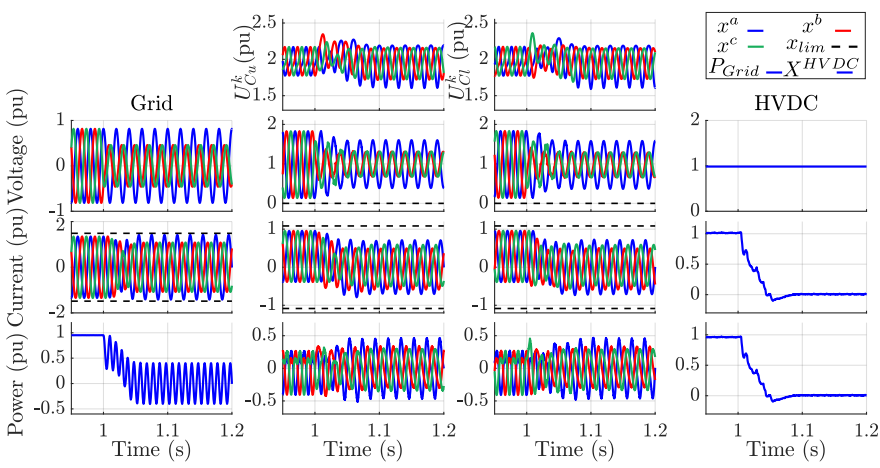

(a) Normal to fault.

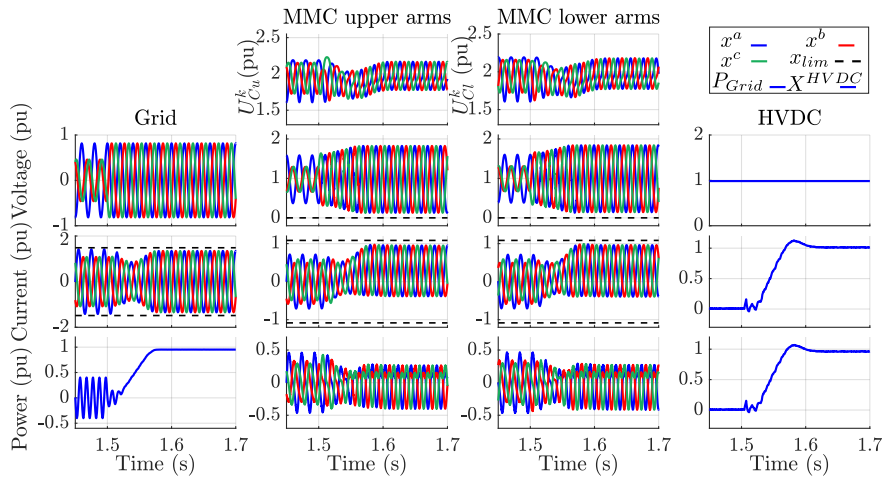

(b) Fault to normal.

Fig. 10. Time-domain waveforms for MMC's main quantities. a) From normal to fault scenario, b) From fault to normal operations.

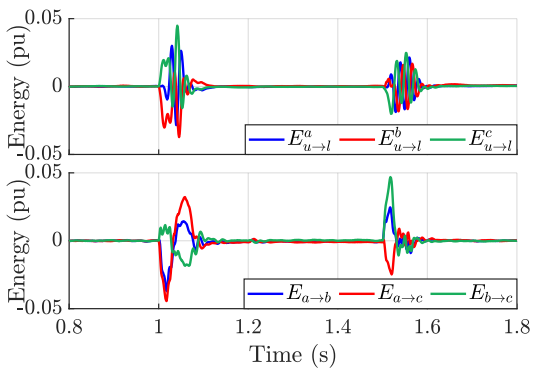

Fig. 11. Internal energy profiles during a type $\mathrm{C}$ fault.

\section{Case IV - HVDC voltage unbalance}

For this case study, the DC and AC grids are considered to be operated under balanced nominal conditions, when the 


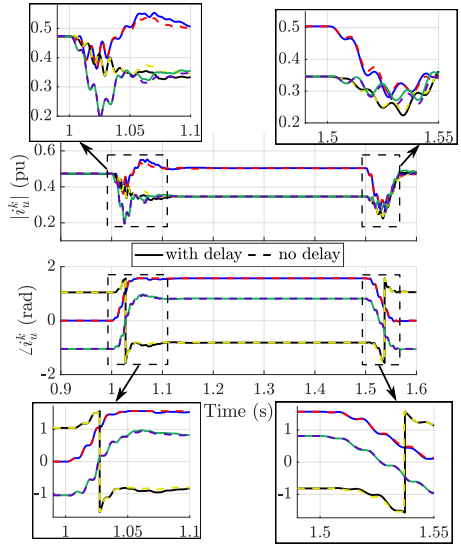

(a) MMC's upper arms currents.

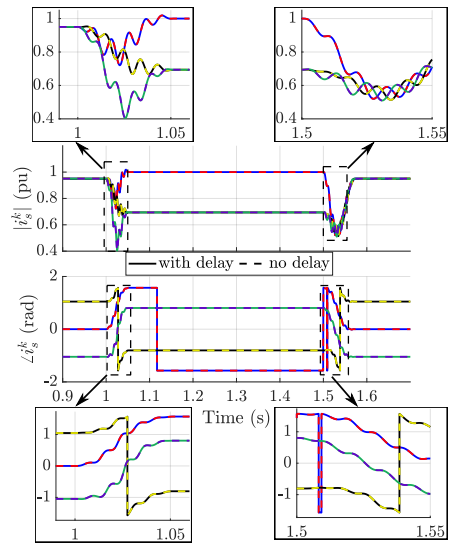

(b) AC grid currents.

Fig. 12. Optimization convergence time impact comparison in the optimal references during SLG fault.

MMC's upper DC pole suffers a voltage drop (from $320 \mathrm{kV}$ to $256 \mathrm{kV}$ ). Such event may arise, for example, in bipolar HVDC multiterminal configurations, where one converter of the system (which is far from the MMC under study) suffers a DC fault. During the fault ride through or blockage stage, as a consequence, other parts of the DC system may observe DC voltage unbalances.

The first thing that can be noted in Fig. 13(a) is that when the DC voltage unbalance happens, the HVDC current injected into the MMC increases in order to maintain the active power level demanded by the AC network. The DC voltage and current deviations are reflected to the internal quantities of the converter rising the current levels circulating through the MMC's arms, which are maintained within the limitations of the converter by the optimization algorithm. Whereas, the arms applied voltages are decreased to their minimum allowed value set to be equal to zero, as the SMs are based on halfbridge topologies. If other SM structures are considered, such as the full-bridge ones, negative voltages could be applied into the arms of the converter; thus, the miminimum allowed arm voltages would be modified to values below zero (e.g. $\left.U_{u, l_{\min }}^{k}<0\right)$. In addition, AC grid currents and, consequently, the AC network power are kept constant throughout the fault due to the optimal control strategy. Similarly, when the fault is cleared, as it is shown in 13(b), the DC side and internal quantities of the converter are set back to normal operating levels, while no change is observed in the AC side.

\section{E. Analysis of the optimization-algorithm convergence time for different $A C$ and $D C$ voltage unbalance conditions}

In this section, the convergence time of the optimization algorithm employed in online applications contemplating different $\mathrm{AC}$ and $\mathrm{DC}$ network voltage conditions are highlighted. The AC grid voltage sags consider $V=0.3 \mathrm{pu}$, whereas for the DC unbalances the same scenarios as Section V-D is used. The purpose of these tests is to confirm that the proposed optimal control strategy can find an optimal solution within each control time step. In Fig. 14, the probability of finding an optimal solution within a specific time-interval is depicted, in which each bar corresponds to a time-interval of

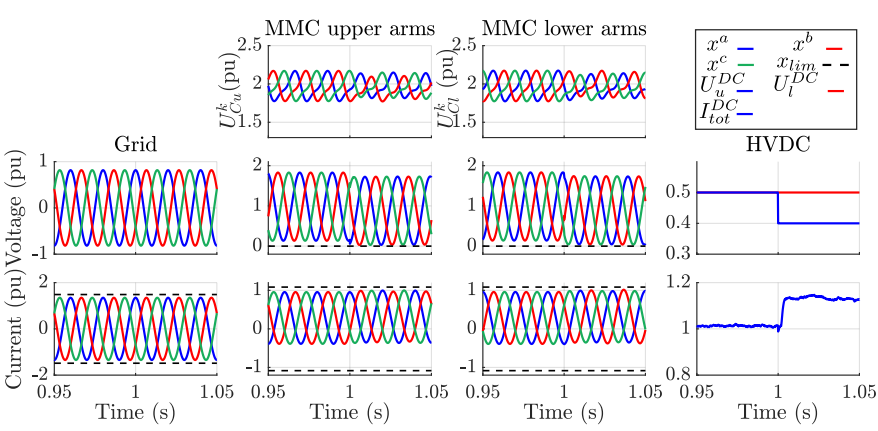

(a) Normal to fault.

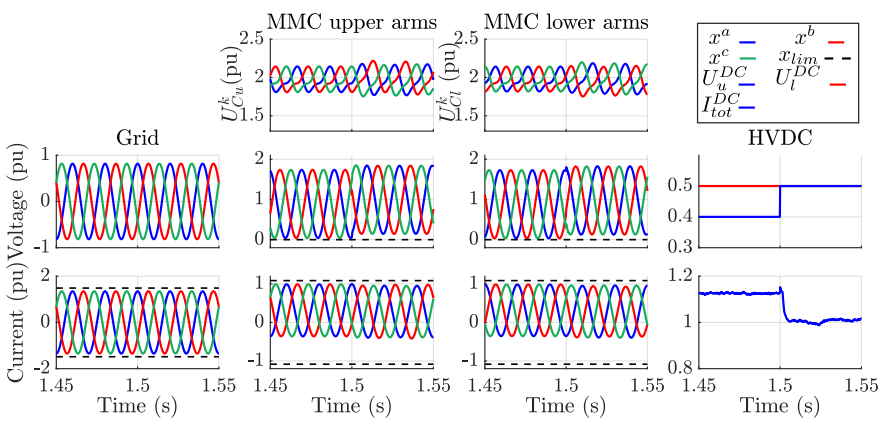

(b) Fault to normal.

Fig. 13. Time-domain waveforms for MMC's main quantities. a) From normal to fault scenario, b) From fault to normal operations.

$500 \mu \mathrm{s}$. As it can be noted, for the several cases analyzed, the probability of occurrence within $2.5 \mathrm{~ms}$ is above $99 \%$. Furthermore, the probability of occurrence within $1.5 \mathrm{~ms}$ is also above $99 \%$ when the converter is operated under normal conditions or during the DC voltage unbalance presented in Section V-D). For the AC grid voltage sags, the balanced case has the highest probability of occurring within $1.5 \mathrm{~ms}$ (more than $80 \%$ ). Finally, for the unbalanced AC network faults, the probability of finding an optimal solution within $1.5 \mathrm{~ms}$ is above $62 \%$.
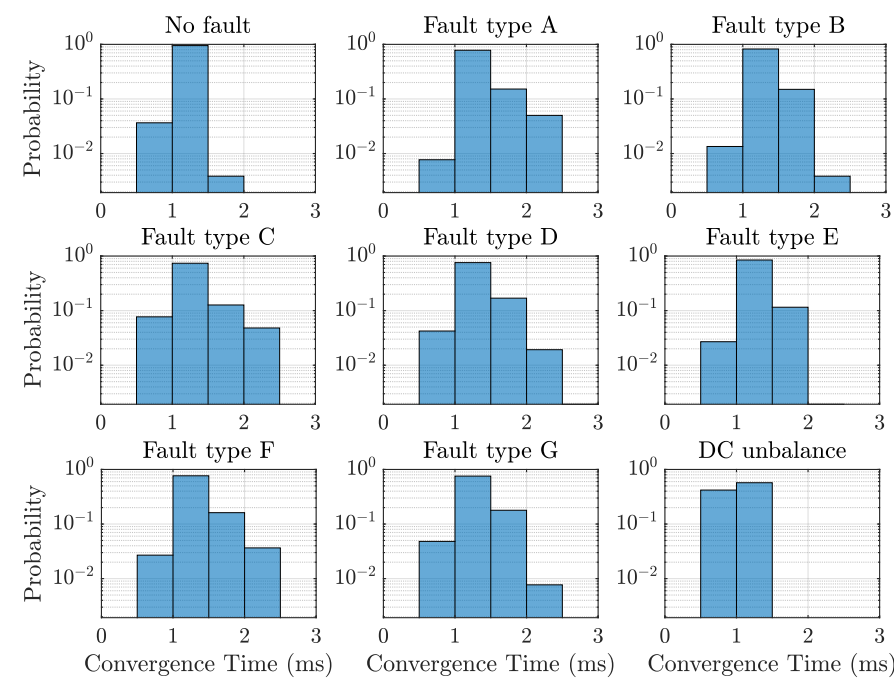

Fig. 14. Convergence time histogram for different AC/DC network conditions.

\section{COMPARISON WITH OTHER CONTROL METHODS}

In this section, the proposed optimization-based reference calculation integrated control is compared with two differ- 
ent MMC control strategies. The first control strategy for comparison is the CCSC (C1) [6], whereas the second one is an energy-based control (C2) [34]. The main difference between strategy $\mathrm{C} 2$ and the proposed method is how the controller references are calculated, as they both have the same controllers with the same gains. When $\mathrm{C} 2$ is employed, the references are calculated based on additive and differential components of the converter, while the proposed approach calculates the references in the natural $a b c$ reference frame through the proposed linearized optimization algorithm. During scenarios where the optimization-algorithm fails to converge, the references provided to the controllers are the same as the ones used in C2 (see Section IV-C).

Both strategies $\mathrm{C} 1$ and $\mathrm{C} 2$ include positive- and negativesequence $\mathrm{AC}$ grid and circulating current controllers and reactive/active AC network currents prioritization based on Cigre B4.70 [5]. The different case studies compare the MMC's responses during different fault and constrained scenarios, assuming the values shown in Table I and that the converter must inject/absorb reactive currents in order to provide voltage support to the network to comply with the grid-code requirements [27]. For all the following case studies, the MMC is firstly considered to be operating in normal $\mathrm{AC}$ network conditions, when at $t=1 \mathrm{~s}$ a SLG fault happens and the fault event is cleared at $t=1.5 \mathrm{~s}$.

\section{A. SLG fault}

In this case study, the performance of the different controllers is analyzed assuming similar current limitations and fault conditions. In Fig. 15, the converter's response during the fault event is shown when the MMC is controlled using strategy C1 [6]. As it can be seen, such strategy cannot maintain the internal quantities of the converter within their design limits and imposes the undesired circulation of $\mathrm{AC}$ current in the DC side of the converter.

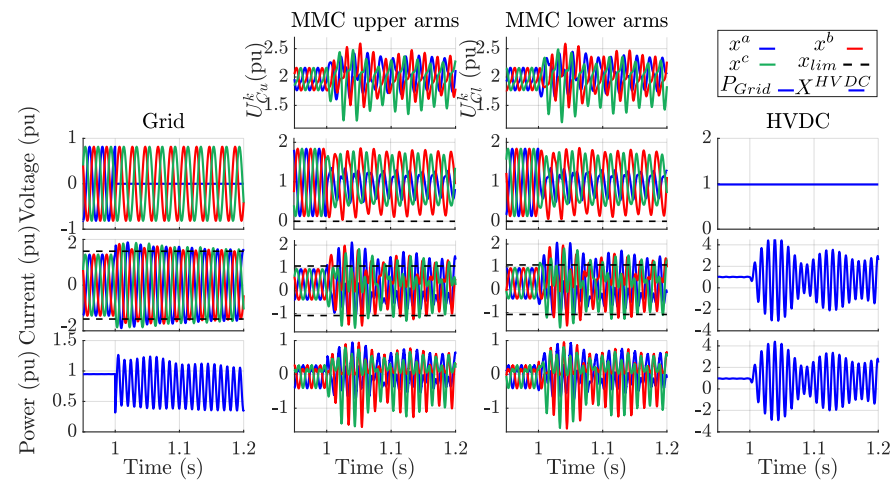

Fig. 15. Time-domain waveforms of the MMC during SLG fault using [6].

In Fig. 16, the MMC's behavior during and after the fault is shown when strategy $\mathrm{C} 2$ is employed. It is clear that strategy $\mathrm{C} 2$ has an improved performance in contrast to $\mathrm{C} 1$, as it avoids the AC oscillations in the DC network while maintaining all the converter's quantities within their limits. Similar conclusions can be drawn when the proposed optimization-based reference calculation integrated control is employed, as it can be noted in Fig. 17. Under such conditions, both $\mathrm{C} 2$ and the optimization-based method present adequate performance and can be potentially employed in a real-application. However, strategy $\mathrm{C} 2$ would require less computational burden (as it does not require an optimization algorithm).

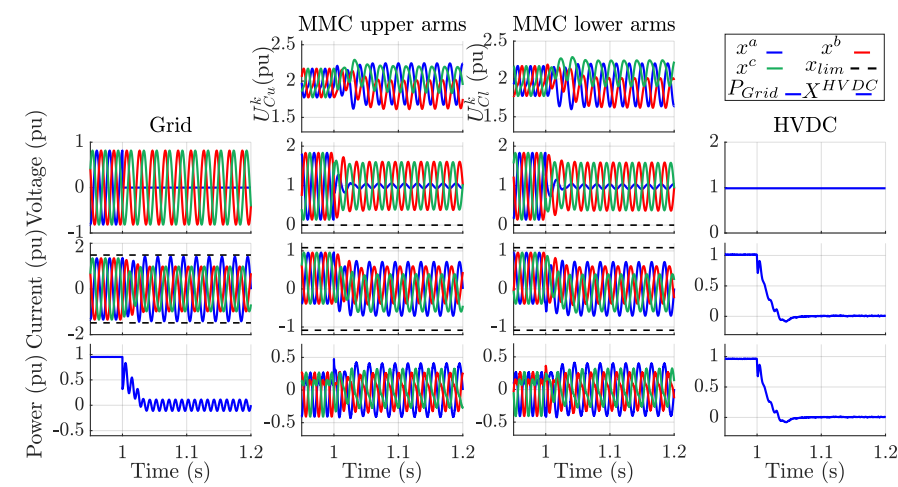

(a) Normal to fault

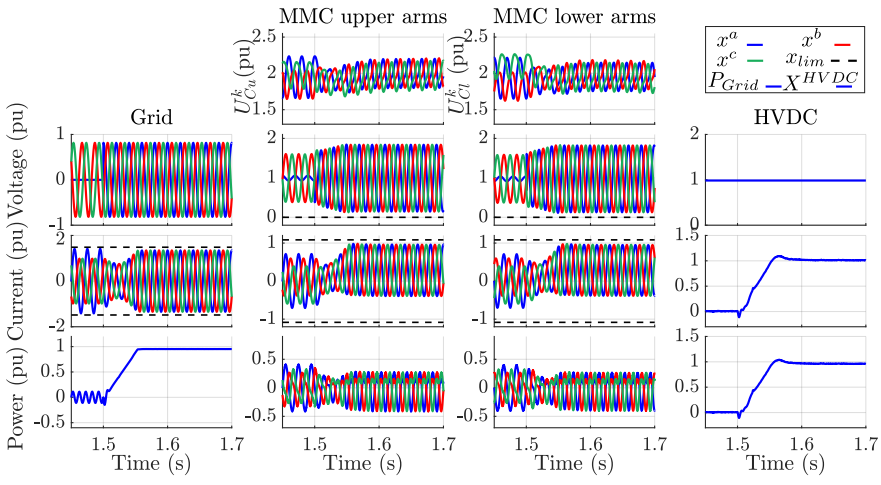

(b) Fault to normal.

Fig. 16. MMC waveforms for control strategy C2 [34] during SLG fault a) Fault is applied to the system and, b) Fault event is cleared.

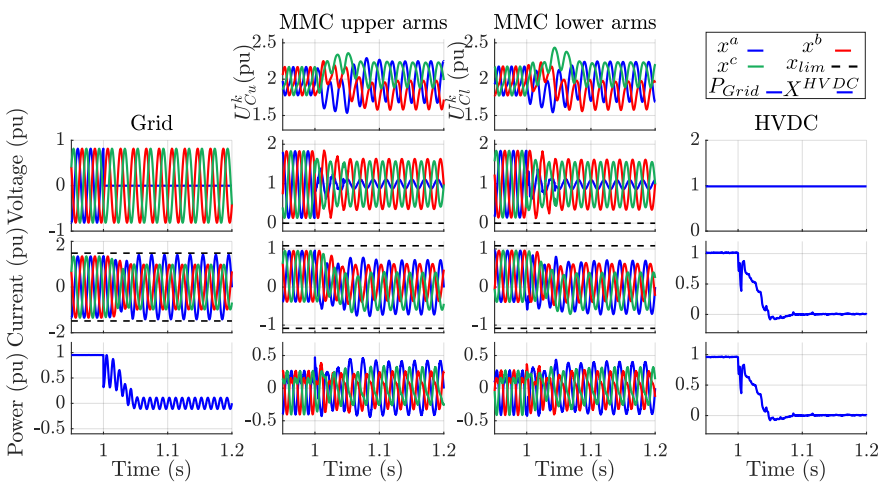

(a) Normal to fault

\section{B. Internal parameters deviations}

For this study, the performance of the proposed optimization-based approach is analyzed and compared with $\mathrm{C} 2$ considering asymmetric MMC's arm impedances during a SLG fault. Strategy C1 has been disregarded, as it presented poor performance in the former analysis (see 

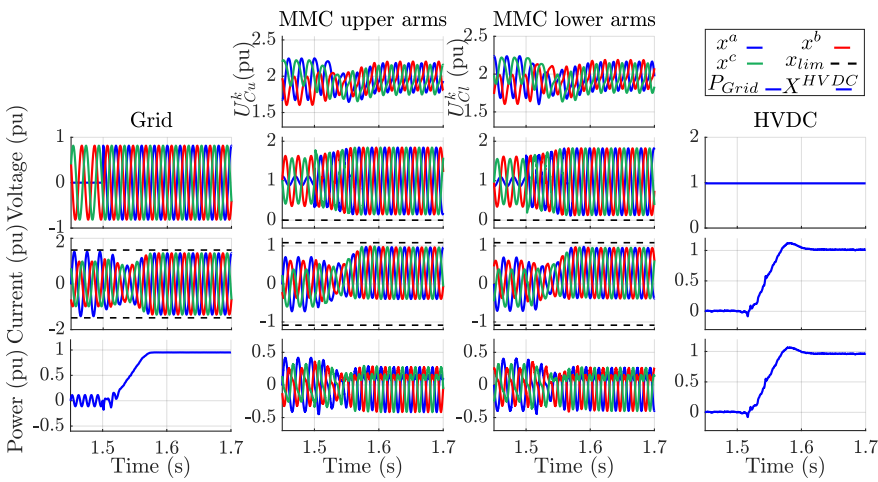

(b) Fault to normal

Fig. 17. MMC waveforms for the proposed optimization-based control during SLG fault a) Fault is applied to the system and, b) Fault event is cleared.

Section VI-A). The reference calculations, the design of the controllers gains and the optimization parameters are maintained constant and they are obtained considering the parameters given in Table I. The arm impedance values employed in this case are highlighted in Table III. Finally, the comparison is done based on the time-domain waveforms of the main quantities of the converter before and after the fault events.

TABLE III

ARM IMPEDANCE VALUES FOR CASE STUDY B

\begin{tabular}{c}
\hline \hline Deviation of $\pm 15 \%$ \\
\hline$\underline{Z}_{u}^{a}=\underline{Z}_{a}-0.15 \underline{Z}_{a}, \underline{Z}_{l}^{a}=\underline{Z}_{a}+0.05 \underline{Z}_{a}$ \\
$\underline{Z}_{u}^{b}=\underline{Z}_{a}-0.10 \underline{Z}_{a}, \underline{Z}_{l}^{b}=\underline{Z}_{a}+0.10 \underline{Z}_{a}$ \\
$\underline{\underline{Z}}_{u}^{c}=\underline{\underline{Z}}_{a}+0.13 \underline{Z}_{a}, \underline{Z}_{l}^{c}=\underline{\underline{Z}}_{a}-0.08 \underline{Z}_{a}$ \\
\hline \hline
\end{tabular}

where $\underline{Z}_{u, l}^{k}$ is the upper and lower arms impedances, with $k \in\{a, b, c\}$.

It can be noted from the top waveforms in Fig. 18a that strategy C2 is unable to maintain the MMC's capacitors with balanced voltage levels even prior to the fault event. Both upper and lower arms' capacitor voltages have different magnitudes, indicating that under asymmetric impedance conditions the control strategy has a poor performance regarding the internal energy of the converter, resulting in voltage unbalances in the SM capacitors. In addition, AC oscillations can be seen in the DC side of the converter. During the fault, the capacitor voltages, especially the lower arm ones, present a severe unbalance profile which may reach the maximum energy deviation allowed in the converter. Furthermore, the $\mathrm{AC}$ oscillation in the DC side are worsen during the fault transient. Once the fault is cleared, as it is shown in Fig. 18b, the aforementioned problems persist.

Next, the same scenario is analyzed assuming that the proposed optimization-based reference calculation integrated control is employed. As it can observed from Fig. 19, the capacitors in the MMC's arms have a balanced voltage profile prior and after the fault. Although there is voltage deviation during the fault, such deviation is maintained constant throughout the fault, which would not lead to a potential trip of the converter. Moreover, the DC side oscillations are completely mitigated, as the optimization algorithm consider such issue in its formulations. Finally, it can be noted that even though

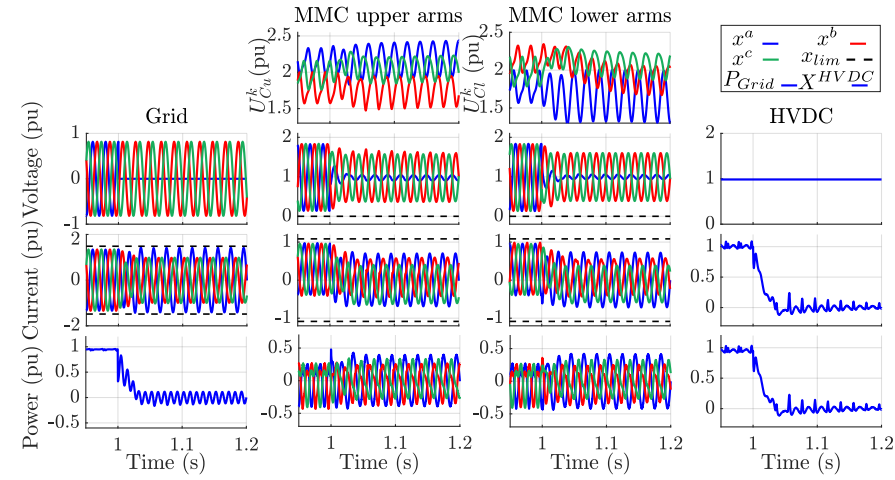

(a) Normal to fault.

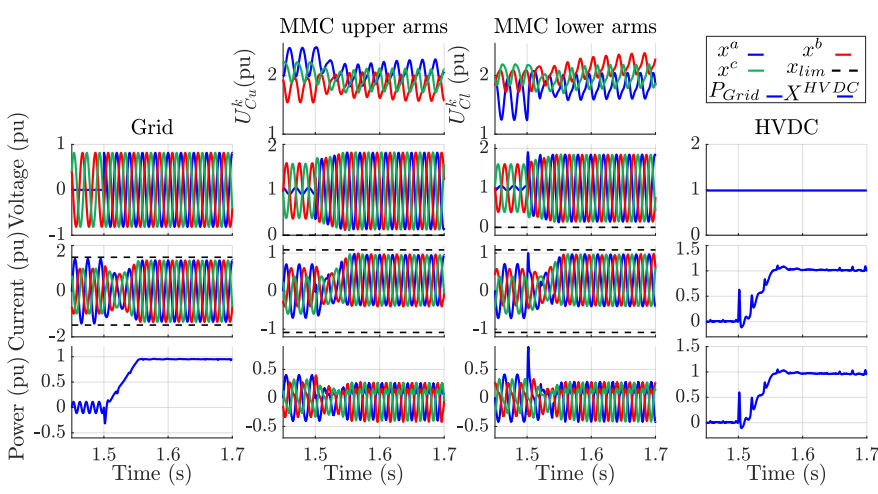

(b) Fault to normal.

Fig. 18. MMC waveforms for control strategy C2 [34] during SLG fault considering unbalanced arm impedances conditions within $\pm 15 \%$ error. a) Fault is applied to the system and, b) Fault event is cleared.

C2 and the proposed method share the same controllers, the references provided by the optimization improved the MMC's performance. Allowing the controllers to mitigate the issues caused by asymmetric arm impedances during a severe unbalanced $\mathrm{AC}$ network voltage condition.

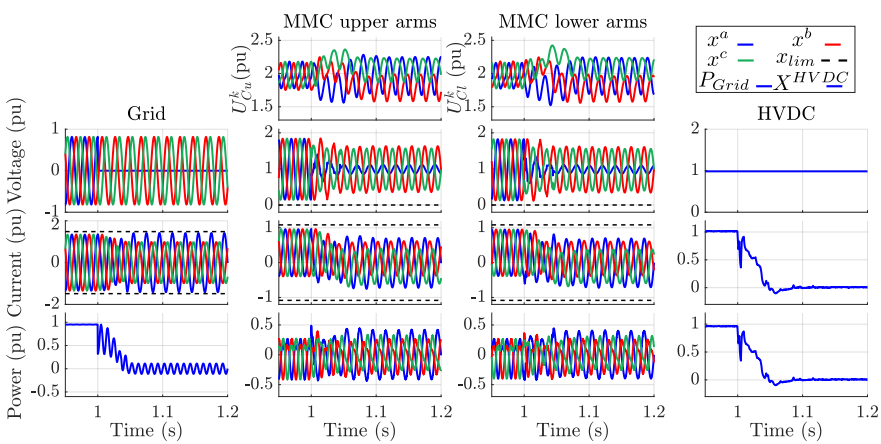

(a) Normal to fault.

\section{Individual arm current limitation}

In addition to the SLG fault, it is also considered the maximum allowed current for the upper arm in phase $c$ is set to be equal to $I_{u_{\max }}^{c}=0.95 \mathrm{pu}$, while for the remaining arms the maximum value is equal to $1.1 \mathrm{pu}$. Such reduction in the maximum allowed current may arise due to cooling or component aging issues. As it was shown in Section VI-A, 


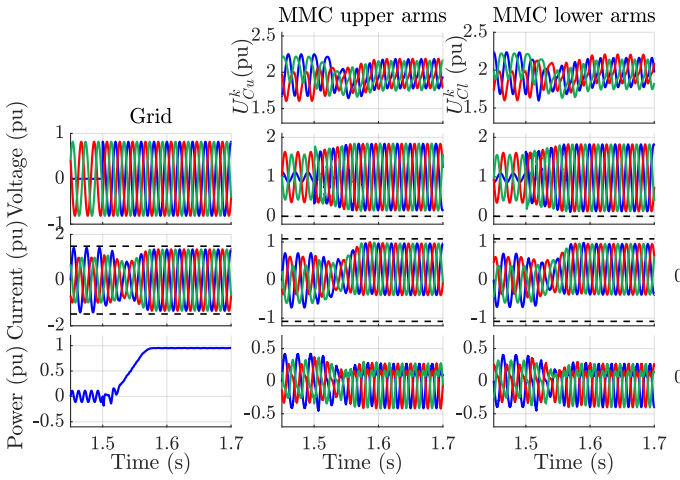

(b) Fault to normal.

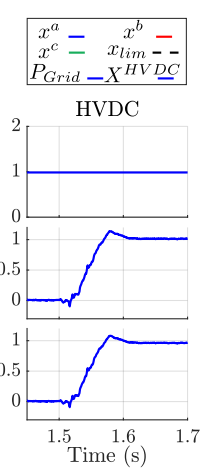

Fig. 19. MMC waveforms for optimization-based control during SLG fault considering unbalanced arm impedances conditions within $\pm 15 \%$ error. a) Fault is applied to the system and, b) Fault event is cleared

during a SLG fault the strategy C1 [6] is unable to properly regulate the converter; thus, such approach is not included in this case study. Although strategy C2 [34] is capable of maintaining the converter operable during the fault, its formulation does not allow to saturate individual arm currents. This happens due to the modelling formulation which uses differential and additive current components. Consequently, all the circulating currents must be reduced equally, leading to a potential converter's performance reduction. Contrarily, as the optimization problem is formulated considering the currents flowing through each individual arm, the proposed method is capable of specifying maximum allowed values for the currents circulating through the converter's arms. For comparison purposes, the saturations employed in the control strategy [34] have been modified. Now, the maximum allowed three-phase DC and AC additive currents are set to $0.95 \mathrm{pu}$. For the proposed method, the maximum allowed current is only reduced for phase $c$ with $I_{u_{\max }}^{c}=0.95 \mathrm{pu}$, whereas the for the remaining the maximum allowed current is set to be equal to $1.1 \mathrm{pu}$. In Figs. 20 and 21, the time-domain waveforms of the converter are presented during the fault event and its clearance.

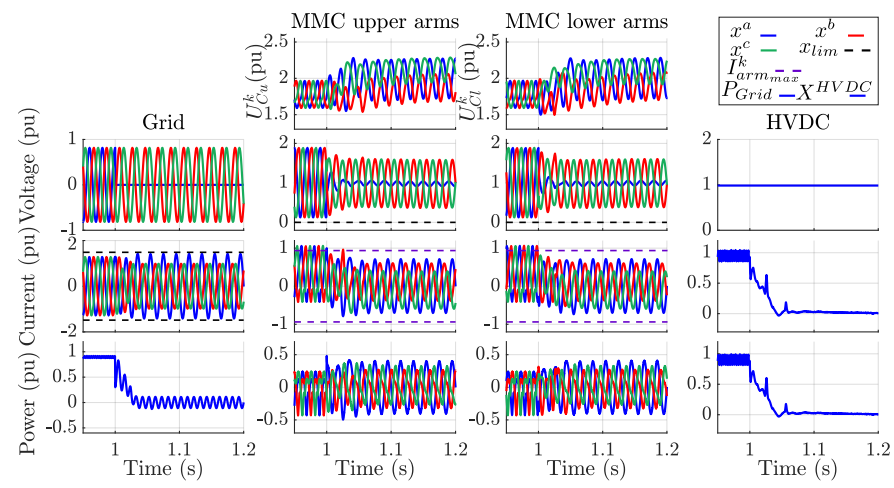

(a) Normal to fault.

Both strategies have similar grid support performance, with $I_{q}^{+}=-1.1 \mathrm{pu}$ and $I_{q}^{-}=0.3414 \mathrm{pu}$. As it can be observed from Fig. 20a, the arm currents prior the fault using C2 are exceeding the imposed limitations and also present highorder frequency oscillations in the DC side of the converter

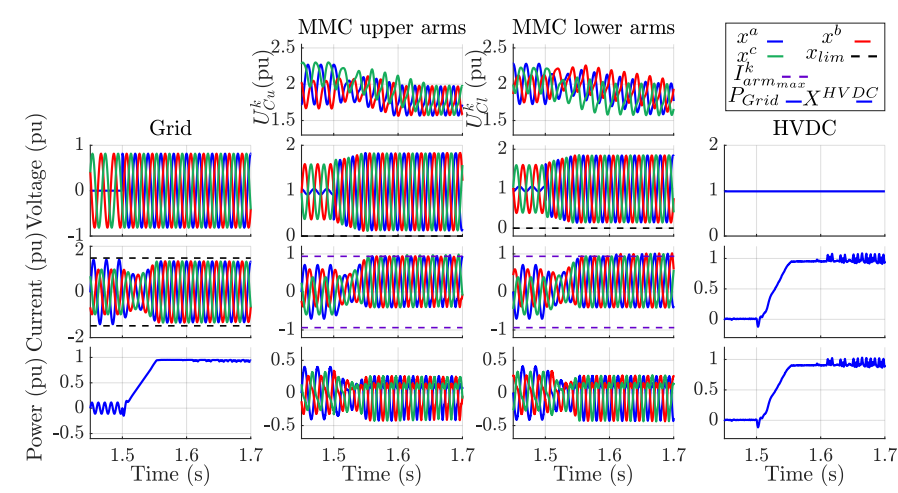

(b) Fault to normal.

Fig. 20. MMC waveforms for control strategy C2 [34] during SLG fault with $I_{\text {arm } \max }^{k}=0.95 \mathrm{pu}$. a) Fault is applied to the system and, b) Fault event is cleared.

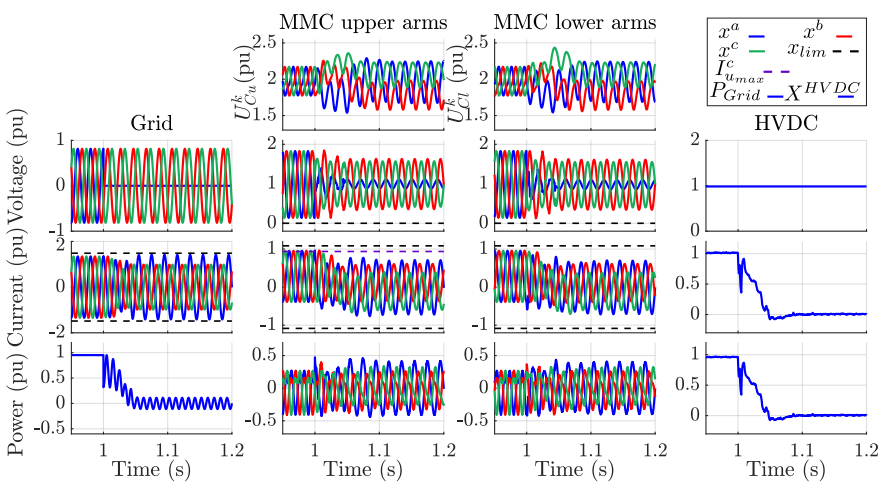

(a) Normal to fault

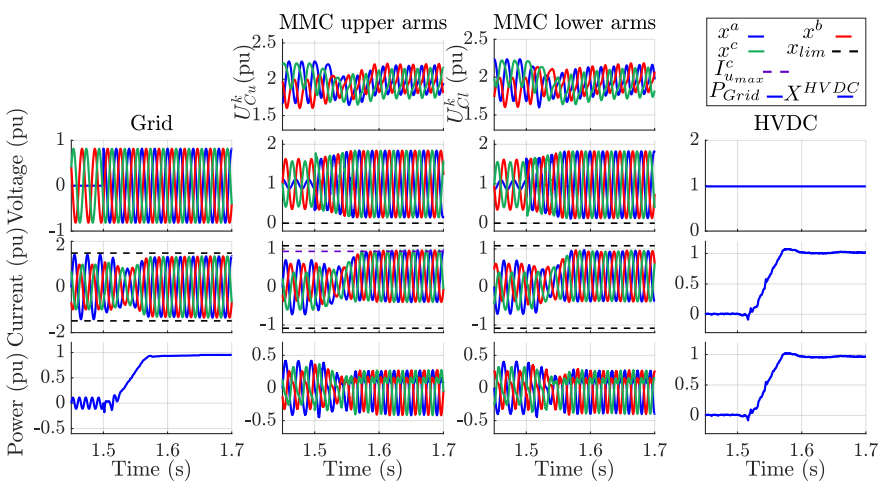

(b) Fault to normal.

Fig. 21. MMC waveforms for optimization-based control during SLG fault considering $I_{u_{\max }}^{c}=0.95$ pu. a) Fault is applied to the system and, b) Fault event is cleared.

due to the harmonic content in the AC additive currents. This control strategy is able to suppress these high order oscillations during the fault, but once the fault is cleared the oscillations can once more be observed in the DC side (see Fig. 20b). On the other hand, the proposed method is capable of avoiding the aforementioned issues throughout the whole operation, keeping the system within the desired limits (see Fig. 21). During normal operation conditions, the upper arm currents present balanced profile and have lower maximum magnitudes compared to the lower arms. Such fact arises due two conditions; firstly, the optimization must maintain 
the power set-points imposed by the operator; secondly, the formulation of the optimization problem, which avoids zero sequence AC currents to flow into the DC side of the converter (observed when strategy C2 [34] was employed).

\section{CONCLUSION}

A real-time optimization-based reference calculation algorithm integrated with a control strategy for modular multilevel converter operating under balanced and unbalanced AC and DC network voltage conditions has been presented. The optimization algorithm has been formulated assuming on a multi-objective problem allowing the prioritization among the positive- and negative-sequence of the active and reactive current component, also considering the internal energy balance of the converter and the AC grid and arm current limitations. Due to the highly nonlinear characteristics of the model which would require nonlinear solver (high computational burden to be solved), the optimization algorithm has been linearized using time-varying approximation. Thus, the linearized optimization problem can be employed in realtime applications, as it can be solved at the same frequency as the control algorithm. The linearized algorithm has been validated through quantitative and time-domain simulations. For all case scenarios analyzed, it has been shown that optimization-based reference calculation integrated with the MMC controllers was capable of maintaining the proper operation of the converter, while attempting to fulfill the gridcode requirements and keeping the internal and $\mathrm{AC}$ currents within their design limitations. Furthermore, the computational viability of the proposed optimization reference generator for real-time implementations was further confirmed based on its convergence time for different network conditions. Finally, the suggested optimization method was compared with other conventional control strategies considering AC network faults and internal parameters deviations. It has been demonstrated that the proposed optimization-based reference calculation integrated control has an improved performance compared to the conventional strategies for all the different scenarios.

\section{REFERENCES}

[1] D. Van Hertem, O. Gomis-Bellmunt, and J. Liang, HVDC Grids: For Offshore and Supergrid of the Future, ser. IEEE Press Series on Power Engineering. Wiley, 2016.

[2] J. Peralta, H. Saad, S. Dennetiere, J. Mahseredjian, and S. Nguefeu, "Detailed and averaged models for a 401-level MMC-HVDC system," IEEE Trans. Power Del., vol. 27, no. 3, pp. 1501-1508, July 2012.

[3] R. Marquardt, "Modular multilevel converter: An universal concept for HVDC-Networks and extended DC-Bus-applications," in The 2010 International Power Electronics Conference - ECCE ASIA -, 2010, pp. 502 507.

[4] M. A. Perez, S. Ceballos, G. Konstantinou, J. Pou, and R. P. Aguilera, "Modular multilevel converters: Recent achievements and challenges," IEEE Open J. Ind. Electron. Soc, pp. 1-1, 2021.

[5] S. Dennetiere, P. Rault, and et. al., "Guide for electromagnetic transient studies involving VSC converters," Cigre, Tech. Rep. WG B4.70, 2021.

[6] Q. Tu, Z. Xu, and L. Xu, "Reduced switching-frequency modulation and circulating current suppression for modular multilevel converters," IEEE Trans. Power Del., vol. 26, no. 3, pp. 2009-2017, 2011.

[7] E. Sánchez-Sánchez, E. Prieto-Araujo, A. Junyent-Ferré, and O. GomisBellmunt, "Analysis of MMC energy-based control structures for VSCHVDC links," IEEE Trans. Emerg. Sel. Topics Power Electron., vol. 6, no. 3, pp. 1065-1076, 2018.
[8] J. Li, G. Konstantinou, H. R. Wickramasinghe, and J. Pou, "Operation and control methods of modular multilevel converters in unbalanced AC grids: A review," IEEE Trans. Emerg. Sel. Topics Power Electron., vol. 7, no. 2, pp. 1258-1271, 2019.

[9] S. Li, X. Wang, Z. Yao, T. Li, and Z. Peng, "Circulating current suppressing strategy for MMC-HVDC based on nonideal proportional resonant controllers under unbalanced grid conditions," IEEE Trans. Power Electron., vol. 30, no. 1, pp. 387-397, 2015.

[10] Z. Ou, G. Wang, and L. Zhang, "Modular multilevel converter control strategy based on arm current control under unbalanced grid condition," IEEE Trans. Power Electrons., vol. 33, no. 5, pp. 3826-3836, 2018.

[11] J. Wang, Y. Tang, and X. Liu, "Arm current balancing control for modular multilevel converters under unbalanced grid conditions," IEEE Trans. Power Electrons., vol. 35, no. 3, pp. 2467-2479, 2020.

[12] S. Cui, H. Lee, J. Jung, Y. Lee, and S. Sul, "A comprehensive AC-side single-line-to-ground fault ride through strategy of an MMC-based HVDC system," IEEE Trans. Emerg. Sel. Topics Power Electron., vol. 6, no. 3, pp. $1021-1031,2018$

[13] J. Lai, X. Yin, X. Yin, Z. Ullah, L. Jiang, and Z. Wang, "Improved comprehensive control of modular multilevel converter under AC/DC grid faults and harmonic operation conditions," IEEE Trans. Power Electrons., vol. 36, no. 6, pp. 6537-6556, 2021.

[14] H. Zhang, M. M. Belhaouane, F. Colas, R. Kadri, F. Gruson, and X. Guillaud, "On comprehensive description and analysis of MMC control design: Simulation and experimental study," IEEE Trans. Power Del., vol. 36, no. 1, pp. 244-253, 2021.

[15] E. Prieto-Araujo, A. Junyent-Ferré, C. Collados-Rodríguez, G. ClarianaColet, and O. Gomis-Bellmunt, "Control design of modular multilevel converters in normal and AC fault conditions for HVDC grids," Electr. Pow. Syst. Res., vol. 152, pp. $424-437,2017$.

[16] G. Bergna-Diaz, J. A. Suul, E. Berne, J. Vannier, and M. Molinas, "Optimal shaping of the MMC circulating currents for preventing AC-side power oscillations from propagating into HVdc grids," IEEE Trans. Emerg. Sel. Topics Power Electron., vol. 7, no. 2, pp. 1015-1030, 2019.

[17] H. Fehr and A. Gensior, "Eigenvalue optimization of the energy-balancing feedback for modular multilevel converters," IEEE Trans. Power Electrons., vol. 34, no. 11, pp. 11 482-11 495, 2019.

[18] M. M. Belhaouane, M. Ayari, X. Guillaud, and N. B. Braiek, "Robust control design of MMC-HVDC systems using multivariable optimal guaranteed cost approach," IEEE Trans. Ind. Appl., vol. 55, no. 3, pp. 2952 2963, 2019.

[19] Z. Gong, X. Wu, P. Dai, and R. Zhu, "Modulated model predictive control for MMC-based active front-end rectifiers under unbalanced grid conditions," IEEE Trans. Ind. Electrons., vol. 66, no. 3, pp. 2398-2409, 2019.

[20] L. Qiu, X. Liu, J. Sun, J. Zhang, J. Ma, and Y. Fang, "Fast finite-set model predictive control for three-phase four-arm active front end modular multilevel converters under unbalanced and distorted network conditions,' IEEE Access, vol. 8, pp. 30 504-30 514, 2020.

[21] ENTSO-E, "Commission regulation (eu) 2016/1447 of 26 august 2016 establishing a network code on requirements for grid connection of high voltage direct current systems and direct current-connected power park modules," p. 65, Aug. 2016.

[22] T. Huang, B. Wang, H. Xie, T. Wu, C. Li, S. Li, J. Hao, and J. Luo, "Research on reactive power control strategy of MMC HVDC converter," in 2020 IEEE 4th Conference on Energy Internet and Energy System Integration (EI2), 2020, pp. 880-884.

[23] X. Chen, G. Irwin, D. Woodford, and A. Gole, "Reactive power control in MMC HVDC system during ac fault," in 12th IET International Conference on AC and DC Power Transmission (ACDC 2016), 2016, pp. 1-5.

[24] M. I. Hossain and M. A. Abido, "Positive-negative sequence current controller for LVRT improvement of wind farms integrated MMC-HVDC network," IEEE Access, vol. 8, pp. 193 314-193339, 2020.

[25] D. W. Spier, E. Prieto-Araujo, J. López-Mestre, and O. Gomis-Bellmunt, "Optimal current reference calculation for mmcs considering converter limitations," IEEE Trans. Power Del., vol. 36, no. 4, pp. 2097-2108, 2021.

[26] National Grid Electricity System Operator Limited, The Grid Code, rev. no. 38, 5 ed., National Grid, Sept 2019.

[27] B. O. del Estado, "Orden TED/749/2020, de 16 de julio, por la que se establecen los requisitos técnicos para la conexión a la red necesarios para la implementación de los códigos de red de conexión,” no. 208, pp. 62 40662 458, Aug. 2020

[28] J. M. Rodriguez-Bernuz, I. Mcinerney, A. Junyent-Ferre, and E. C. Kerrigan, "Design of a linear time-varying model predictive control energy regulator for grid-tied VSCs," IEEE Trans. Energy Convers., pp. 1-1, 2021.

[29] J. Rodriguez-Bernuz and A. Junyent-Ferré, "Operating region extension of a modular multilevel converter using model predictive control: A single phase analysis," IEEE Trans. Power Del., vol. 35, no. 1, pp. 171-182, 2020.

[30] R. Fletcher, Practical Methods of Optimization, 2nd ed. New York, NY, USA: John Wiley \& Sons, 1987. 
[31] B. Stellato, G. Banjac, P. Goulart, A. Bemporad, and S. Boyd, "OSQP: an operator splitting solver for quadratic programs," Mathematical Programming Computation, vol. 12, no. 4, pp. 637-672, 2020.

[32] M. Bollen and L. Zhang, "Different methods for classification of threephase unbalanced voltage dips due to faults," Electr. Power Syst. Res., vol. 66 , no. 1 , pp. $59-69,2003$

[33] H. Akagi, E. H. Watanabe, and M. Aredes, "Instantaneous power theory and applications to power conditioning," 2007.

[34] D. W. Spier, E. Prieto-Araujo, J. López-Mestre, and O. Gomis-Bellmunt, "Improved current reference calculation for MMCs internal energy balancing control," IEEE Trans. Power Del., pp. 1-1, 2021.

[35] ENTSO-E, "ENTSO-E network code on requirements for grid connection of generators," (accessed on 2020-10-29). [Online]. Available: https://eur-lex.europa.eu/legalcontent/EN/TXT/PDF/?uri=CELEX:32016R0631\&from=EN

[36] J. Xu, C. Zhao, W. Liu, and C. Guo, "Accelerated model of modular multilevel converters in PSCAD/EMTDC," IEEE Transactions on Power Delivery, vol. 28, pp. 129-136, 2013.

[37] Q. Tu and Z. Xu, "Impact of sampling frequency on harmonic distortion for modular multilevel converter," IEEE Transactions on Power Delivery, vol. 26, pp. 298-306, 2011. 